\title{
RADIAL SYMMETRY OF SOLUTIONS TO ANISOTROPIC AND WEIGHTED DIFFUSION EQUATIONS WITH DISCONTINUOUS NONLINEARITIES
}

\author{
SERENA DIPIERRO, GIORGIO POGGESI, AND ENRICO VALDINOCI
}

AbSTRACT. For $1<p<\infty$, we prove radial symmetry for bounded nonnegative solutions of

$$
\begin{cases}-\operatorname{div}\left\{w(x) H(\nabla u)^{p-1} \nabla_{\xi} H(\nabla u)\right\}=f(u) w(x) & \text { in } \Sigma \cap \Omega, \\ u=0 & \text { on } \Gamma_{0}, \\ \left\langle\nabla_{\xi} H(\nabla u), \nu\right\rangle=0 & \text { on } \Gamma_{1} \backslash\{0\},\end{cases}
$$

where $\Omega$ is a Wulff ball, $\Sigma$ is a convex cone with vertex at the center of $\Omega, \Gamma_{0}:=\Sigma \cap \partial \Omega, \Gamma_{1}:=\partial \Sigma \cap \Omega, H$ is a norm, $w$ is a given weight and $f$ is a possibly discontinuous nonnegative nonlinearity.

Given the anisotropic setting that we deal with, the term "radial" is understood in the Finsler framework, that is, the function $u$ is radial if there exists a point $x$ such that $u$ is constant on the Wulff shapes centered at $x$.

When $\Sigma=\mathbb{R}^{N}$, J. Serra obtained the symmetry result in the isotropic unweighted setting (i.e., when $H(\xi) \equiv$ $|\xi|$ and $w \equiv 1)$. In this case we provide the extension of his result to the anisotropic setting. This provides a generalization to the anisotropic setting of a celebrated result due to Gidas-Ni-Nirenberg and such a generalization is new even for $p=2$ whenever $N>2$.

When $\Sigma \subsetneq \mathbb{R}^{N}$ the results presented are new even in the isotropic and unweighted setting (i.e., when $H$ is the Euclidean norm and $w \equiv 1$ ) whenever $2 \neq p \neq N$. Even for the previously known case of unweighted isotropic setting with $p=2$ and $\Sigma \subsetneq \mathbb{R}^{N}$, the present paper provides an approach to the problem by exploiting integral (in)equalities which is new for $N>2$ : this complements the corresponding symmetry result obtained via the moving planes method by Berestycki-Pacella.

The results obtained in the isotropic and weighted setting (i.e., with $w \not \equiv 1$ ) are new for any $p$.

\section{INTRODUCTION}

Since the classical works of Aleksandrov [A], Serrin [ [S] and Gidas-Ni-Nirenberg [GNN], an intensively studied topic in the theory of partial differential equations and the calculus of variations focuses on the radial symmetry of solutions under suitable assumptions on the equation under consideration, on the boundary conditions and/or on the domain.

In this paper we consider the Finsler framework of a nonlinear anisotropic equation in divergence form. The setting taken into account is quite general, since it includes nonlinear operators of $p$-Laplace type, possibly with weights. The domain considered is obtained from the intersection of a Wulff ball and a cone $\Sigma \subseteq \mathbb{R}^{N}$ with vertex at its center. The results provided are of radial symmetry type, where, given the possible anisotropy of the ambient space, the term "radial" is understood in the sense that a function $u$ is radial if there exists a point $x$ such that $u$ is constant on the Wulff shapes centered at $x$ (see Section 2 for the detailed mathematical setting).

These results will be obtained under suitable homogeneity or concavity assumptions on the weights $w$ and suitable arithmetic relations between the dimension of the ambient space, the homogeneous exponent of the weight and the homogeneity of the nonlinear operator. A pivotal step in our analysis consists in proving that the level sets of the solution are isoperimetric (and additionally the anisotropic norm of the gradient of the solution is constant along these level sets). This cornerstone result will be stated in detail in Theorem 1.1 below and then combined with a series of isoperimetric inequalities to establish that the level sets are Wulff shapes: this, together with the additional information on the constancy of the anisotropic norm of the gradient of the solution, establishes the radial symmetry of the solution. Depending on technical conditions on the (an)isotropy of the ambient space and on the weights, these radial symmetry results will be detailed in Theorems 1.4, 1.5 and 1.6.

The arguments that we use are inspired by those of J. Serra in $[\mathrm{Se}$, which in turn were inspired by the classical paper of P.-L. Lions $[\mathrm{L}$ in which the symmetry was obtained in the Euclidean case with $p=N=2$, $\Sigma=\mathbb{R}^{N}$ and $w \equiv 1$. We remark that the main results in $[\mathrm{L}$, focusing on elliptic semilinear equations in the plane, can be seen as a counterpart of those in GNN], in the sense that the results in [L weaken the smoothness assumptions on the source term and on the solution with respect to the setting in [GNN], at

2020 Mathematics Subject Classification. 35B06.

Key words and phrases. Symmetry, Convex cones, Weighted anisotropic isoperimetric inequalities. 
the expense of restricting to positive nonlinearities and to dimension two only. Interestingly, the method in $[\mathrm{L}$, being based on integral (in)equalities, is conceptually different from the moving plane technique used in GNN and, in a sense, it is more related, apart from several important structural differences, to the approach to radial symmetry inhaugurated by Weinberger in $\mathrm{W}$ : see also, e.g., $\mathrm{PSc}, \mathrm{Re}, \mathrm{GL}, \mathrm{FGK}, \mathrm{FK}$, BNST, MP, MP2, MP3, GS, EP, Po, CS, WX, BC, GX, DPV, PT, PT2, CG, CiR, CFR, for related problems and ramifications.

The method of $[\mathrm{L}$ has been extended by Kesavan-Pacella to the $N$-dimensional Euclidean setting in presence of the $p$-Laplacian operator in the case $p=N$, see $[\mathrm{KP}$. In turn, the techniques of [KP have triggered the research in the anisotropic case (with $\Sigma=\mathbb{R}^{N}$ and $p=N$ ), which was carried out by BelloniFerone-Kawohl in [BFK]. The extension of the method to the case $p \neq N$ is due to J. Serra ([Se]), who obtained it in the isotropic setting (with $\Sigma=\mathbb{R}^{N}$ ).

In $[\mathrm{BP}$, by using the moving planes method, Berestycki-Pacella obtained radial symmetry in spherical convex cones (in the isotropic unweighted setting), when $p=2$ and $f$ is Lipschitz. To the authors' knowledge, symmetry results in spherical convex cones with $\Sigma \subsetneq \mathbb{R}^{N}$ were considered only in the unweighted isotropic setting in $[\mathrm{BP}]($ for $p=2$ ) and $[\mathrm{KP}$ ( for $p=N)$.

See also [DFM] and the references therein for further works on symmetry problems for nonlinear equations.

We point out that, while our method is general enough to work in the anisotropic weighted setting, the results offered here are new also in some more classical cases (such as those stated in Theorems 1.4, [1.5 and 1.6). Moreover, thanks to the available characterizations of the isoperimetric sets, the conclusions obtained in these cases are stronger and take a less technical form. As an introductory example, one of the results that we obtain here (see Theorem [1.5) goes as follows.

Let $\Omega$ be a Wulff ball in $\mathbb{R}^{N}, N \geqslant 2$, and let $1<p<\infty$. Assume that $f \in L_{\mathrm{loc}}^{\infty}([0, \infty))$ is nonnegative. Let $u \in C^{1}(\bar{\Omega})$ be a weak solution of

$$
\begin{cases}-\operatorname{div}\left\{H(\nabla u)^{p-1} \nabla_{\xi} H(\nabla u)\right\}=f(u) & \text { in } \Omega, \\ u \geqslant 0 & \text { in } \Omega, \\ u=0 & \text { on } \partial \Omega\end{cases}
$$

Assume that either

$$
p \geqslant N
$$

or

$$
p<N \quad \text { and, for some nonincreasing function } \phi \geqslant 0 \text {, we have } \phi \leqslant f \leqslant \frac{N p}{N-p} \phi .
$$

Then, $u$ is a radially symmetric and radially nonincreasing function. Moreover,

and

$$
u \text { is radially strictly decreasing on }\left\{0<u<\max _{\bar{\Omega}} u\right\} \text {, }
$$

$$
\left\{0<u<\max _{\bar{\Omega}} u\right\} \quad \text { is a Wulff annulus or a punctured Wulff ball. }
$$

Let us now go into the technical framework of this paper, to present the results obtained in their full generality.

Let $\Sigma$ be an open cone in $\mathbb{R}^{N}$ with vertex at the origin, i.e.,

$$
\Sigma:=\{t x: x \in \omega, t \in(0, \infty)\},
$$

for some domain $\omega \subseteq \mathbb{S}^{N-1}$. We stress that the possibility $\omega=\mathbb{S}^{N-1}$ (which implies $\Sigma=\mathbb{R}^{N}$ ) is allowed throughout the paper. When $\omega \subsetneq \mathbb{S}^{N-1}$, we assume $\Sigma$ to be convex and denote by $\nu$ its outward unit normal (which is defined almost everywhere on $\partial \Sigma$ ).

Let $\Omega \subset \mathbb{R}^{N}$ be a bounded domain, and define

$$
\Gamma_{0}:=\Sigma \cap \partial \Omega \quad \text { and } \quad \Gamma_{1}:=\partial \Sigma \cap \Omega .
$$

Furthermore, we endow $\mathbb{R}^{N}$ with a norm $H: \mathbb{R}^{N} \rightarrow \mathbb{R}$ such that:

$$
\begin{aligned}
& H \text { is convex; } \\
& H(\xi) \geqslant 0 \text { for } \xi \in \mathbb{R}^{N} \text { and } H(\xi)=0 \text { if and only if } \xi=0 ; \\
& H(t \xi)=|t| H(\xi) \text { for } \xi \in \mathbb{R}^{N} \text { and } t \in \mathbb{R} .
\end{aligned}
$$

We also define

$$
B^{H}:=\left\{\xi \in \mathbb{R}^{N}: H(\xi)<1\right\}
$$


Throughout the paper, we assume $H \in C^{2}\left(\mathbb{R}^{N} \backslash\{0\}\right)$ to be uniformly elliptic. We say that $H \in C^{2}\left(\mathbb{R}^{N} \backslash\{0\}\right)$ is uniformly elliptic if the ball $B^{H}$ is uniformly convex, i.e., such that the principal curvatures of the boundary of $B^{H}$ are bounded away from zero. This is a standard assumption on $H$ in order to obtain some regularity of the solutions, using or adapting the elliptic regularity theory (see, e.g., also [BC, $\overline{C F V}]$ ).

In this setting, we consider solutions of

$$
\begin{cases}-\operatorname{div}\left\{w(x) H(\nabla u)^{p-1} \nabla_{\xi} H(\nabla u)\right\}=f(u) w(x) & \text { in } \Sigma \cap \Omega, \\ u \geqslant 0 & \text { in } \Sigma \cap \Omega, \\ u=0 & \text { on } \Gamma_{0}, \\ \left\langle\nabla_{\xi} H(\nabla u), \nu\right\rangle=0 & \text { on } \Gamma_{1} \backslash\{0\} .\end{cases}
$$

In the case $\Sigma=\mathbb{R}^{N}$, we have $\Gamma_{1}=\varnothing$ and the last condition in (1.5) is trivially satisfied. In this case, (1.5) simply becomes

$$
\begin{cases}-\operatorname{div}\left\{w(x) H(\nabla u)^{p-1} \nabla_{\xi} H(\nabla u)\right\}=f(u) w(x) & \text { in } \Omega, \\ u \geqslant 0 & \text { in } \Omega, \\ u=0 & \text { on } \partial \Omega .\end{cases}
$$

In our main results, the assumptions on the weight $w$ are the following]: there exists $\lambda \geqslant 0$ such that

$$
w: \bar{\Sigma} \rightarrow[0,+\infty) \text { is a continuous nonnegative function positively homogeneous of degree } \lambda,
$$$$
w^{1 / \lambda} \text { is concave in } \Sigma \text { (in the case } \lambda>0 \text { ), }
$$

We notice that when $\lambda>0$, the concavity assumption on $w^{1 / \lambda}$ automatically gives that $w$ is positive in $\Sigma$.

To state our main theorem in this setting, we recall the following notation. We identify the dual space of $\mathbb{R}^{N}$ with $\mathbb{R}^{N}$ itself via the scalar product $\langle\cdot, \cdot\rangle$. Accordingly, given the norm $H$ satisfying (1.1), (1.2) and (1.3), the space $\mathbb{R}^{N}$ turns out to be endowed with the dual norm $H_{0}$, that is the polar function defined by

$$
H_{0}(x)=\sup _{\xi \neq 0} \frac{\langle x, \xi\rangle}{H(\xi)} \quad \text { for } \quad x \in \mathbb{R}^{N} .
$$

Notice that $H$ results to be the support function of the unitary Wulff ball

$$
B^{H_{0}}:=\left\{x \in \mathbb{R}^{N}: H_{0}(x)<1\right\}
$$

of $H$ centered at the origin (see $[\mathrm{CrM}]$ and $\left[\mathrm{Sc}\right.$, Section 1.7]) and, in turn, $H_{0}$ is the support function of $B^{H}$, defined in (1.4). To ease notation, the unitary Wulff ball (of $H$ ) $B^{H_{0}}$ centered at the origin will be denoted simply by $B$. Similarly, for $r>0, B_{r}$ will denote the Wulff ball (of $H$ ) of radius $r$ centered at the origin, i.e.,

$$
B_{r}:=B_{r}^{H_{0}}:=\left\{x \in \mathbb{R}^{N}: H_{0}(x)<r\right\} .
$$

Furthermore, for a set of finite perimeter $E$, we define the weighted anisotropic perimeter of $E$ in $\Sigma$ as follows

$$
P_{w, H}(E ; \Sigma):=\int_{\Sigma \cap \partial^{*} E} H(\nu) w d \mathcal{H}^{N-1},
$$

being $\partial^{*} E$ the reduced boundary of $E$ and $\nu$ its outer (measure theoretical) unit normal vector. Also, for a measurable set $E \subset \Sigma$, we denote by $w(E)$ the weighted volume of $E$, namely

$$
w(E):=\int_{E} w d \mathcal{H}^{N} .
$$

In the unweighted case (i.e., when $w \equiv 1), w(E)$ agrees with $\mathcal{H}^{N}(E)$.

With this notation, our main theorem is the following:

\footnotetext{
${ }^{1}$ Using the recent results in $\left[\right.$ ], assumption 1.8 may be weakened by requiring that, when $\lambda>0$, either $w$ or $w^{1 / \lambda}$ is concave, or, equivalently, that either $\lambda \in(0,1)$ and $w$ is concave or $\lambda \in[1,+\infty)$ and $w^{1 / \lambda}$ is concave. This generalization is accomplished by using, when $w$ is concave, the isoperimetric inequality in Corollary 0.14 (see also Remark 0.15) of [I] instead of the one by CRS recalled here in Theorem 4.1 Strictly speaking, the setting in II is isotropic, but the arguments introduced there have the potential to be generalized to obtain analogous results in the anisotropic setting as well. Counterexamples to the isoperimetric inequality are also given in Corollary 0.12 and Remark 0.13 of [I]

We also point out that the constant in [I] (which is in terms of the volume ratio) improves in several cases the previously obtained ones.
} 
Theorem 1.1. Let $\Omega$ be a Wulff ball in $\mathbb{R}^{N}$ centered at $0, N \geqslant 2$, and let $1<p<\infty$. Assume that $f \in L_{\mathrm{loc}}^{\infty}([0, \infty))$ is nonnegative and the weight $w$ satisfies (1.7), (1.8), and (1.9).

Let $u \in C^{1}\left((\Sigma \cap \Omega) \cup \Gamma_{0} \cup\left(\Gamma_{1} \backslash\{0\}\right)\right) \cap W^{1, \infty}(\Sigma \cap \Omega)$ be a solution of (1.5) in the weak sense. Set

$$
D:=N+\lambda,
$$

where $\lambda$ is that appearing in (1.7). Assume that either

$$
p \geqslant D
$$

or

$$
p<D \quad \text { and, for some nonincreasing function } \phi \geqslant 0 \text {, we have } \phi \leqslant f \leqslant \frac{D p}{D-p} \phi \text {. }
$$

Let also

$$
M:=\sup _{\Sigma \cap \Omega} u,
$$

Then, for a.e. $t \in(0, M)$ the following two conditions are verified:

(i) $\{u>t\}$ satisfies

$$
\frac{P_{w, H}(\{u>t\} ; \Sigma)}{w(\Sigma \cap\{u>t\})^{\frac{D-1}{D}}}=\frac{P_{w, H}(B ; \Sigma)}{w(\Sigma \cap B)^{\frac{D-1}{D}}},
$$

(ii) $H(\nabla u)$ is constant on $\{u=t\}$.

We remark that, in the setting of Theorem 1.1. since $u \in W^{1, \infty}(\Sigma \cap \Omega)$ and $\Sigma \cap \Omega$ is convex, the Sobolev embedding theorem (see also [H], Theorem 4.1]) ensures that $u \in C^{0,1}(\overline{\Sigma \cap \Omega}) \subset C^{0}(\overline{\Sigma \cap \Omega})$, and hence an equivalent definition in (1.15) is to set

$$
M:=\frac{\max }{\Sigma \cap \Omega} u
$$

We also point out that formula (1.16) says that the sets $\{u>t\}$ satisfy the equality in the weighted anisotropic isoperimetric inequality in cones established in CRS. This will be recalled in details in Theorem 4.1

Remark 1.2 (On the regularity of the solutions). Concerning the regularity assumptions on $u$ in Theorem 1.1 we point out that the main hypothesis is that $u$ belongs to $W^{1, \infty}(\Sigma \cap \Omega)$, while the additional smoothness assumptions may be dropped. Moreover, when $\Sigma=\mathbb{R}^{N}$, also the assumption $u \in W^{1, \infty}(\Sigma \cap \Omega)$ could be dropped and replaced just by the assumption that $u$ is bounded.

Indeed, in the case where $\Sigma=\mathbb{R}^{N}$, whenever $\Omega$ is a $C^{1, \gamma}$ domain, with $0<\gamma \leqslant 1$, then [Li], Theorem 1] ensures that bounded solutions to (1.6) are automatically $C^{1, \gamma}(\bar{\Omega})$, for some $0<\gamma \leqslant 1$. Notice that, our regularity assumption on $H$ ensures that $\Omega$ in Theorem 1.1. which is a Wulff ball, is of class $C^{2}$. Thus, in the case when $\Sigma=\mathbb{R}^{N}$, all the regularity assumptions on $u$ in Theorem 1.1 are satisfied by any bounded solution.

When $\Sigma \subsetneq \mathbb{R}^{N}$, we have to consider the mixed boundary value problem (1.5). For such a problem, regularity up to the (whole) boundary is a delicate issue, and it strongly depends on how $\Sigma$ and $\Omega$ intersect. Nevertheless, [Li, Theorem 1] still ensures the $C^{1, \gamma}$-regularity (of bounded solutions) up to $\Gamma_{0}$; the $C^{1, \gamma_{-}}$ regularity up to $\Gamma_{1} \backslash\{0\}$ could be obtained by [Li, Theorem 2], if we further assume that $\Gamma_{1} \backslash\{0\}$ is of class $C^{1, \gamma}$, with $0<\gamma \leqslant 1$, and $w$ satisfy the additional requirement

$$
w \text { is positive and Hölder continuous on } \overline{\Sigma \cap \Omega} \backslash\{0\} \text {. }
$$

We stress that our results are presented without these additional assumptions: that is, we do not require (1.17) and we only ask $\Sigma$ to be convex (with no need of the additional assumption that $\Gamma_{1} \backslash\{0\}$ is of class $C^{1, \gamma}$ ).

Essentially, the main regularity assumption that we impose on $u$ in Theorem 1.1 (when $\Sigma \subsetneq \mathbb{R}^{N}$ ) is that $u$ belongs to $W^{1, \infty}(\Sigma \cap \Omega)$. This is needed in order to ensure the validity of certain integral identities, such as the Pohozaev-type identity (3.17), by using an approximation argument. We notice that, when $H$ is the Euclidean norm and $w \equiv 1, \mathbf{C M}$, Theorem 1.2] guarantees the $W^{1, \infty}$ regularity up to $\Gamma_{1}$ (including the vertex). As pointed out in $\left[\mathrm{PT}\right.$, the assumption $u \in W^{1, \infty}(\Sigma \cap \Omega)$ can be seen as a gluing condition between the cone and $\Gamma_{0}$. Related to this, we mention that $\left[\mathrm{PT}\right.$, Proposition 6.1] guarantees $C^{2}(\bar{\Omega} \backslash\{0\})$-regularity for weak solutions of

$$
\begin{cases}-\Delta u=1 & \text { in } \Sigma \cap \Omega, \\ u=0 & \text { on } \Gamma_{0}, \\ \langle\nabla u, \nu\rangle=0 & \text { on } \Gamma_{1} \backslash\{0\},\end{cases}
$$

in general domains $\Omega$, whenever $\Gamma_{0}$ and $\Gamma_{1}$ intersect orthogonally. 
Remark 1.3 (On the weights). The assumptions (1.7), (1.8) and (1.9) on $w$ guarantee the validity of the weighted anisotropic isoperimetric inequality (4.1) in convex cones, obtained in [CRS]. The homogeneity assumption on $w$ contained in (1.7) is also used to obtain the Pohozaev-type identity in Lemma 3.4 .

Various examples of weights satisfying (1.7), (1.8) and (1.9) are provided in CRS. We point out that all the weights provided in those examples also satisfy assumption (1.17), up to substituting $\Sigma$ with a smaller cone $\Sigma^{\prime} \subset \Sigma$ (see also item (iii) at page 2983 in [CRS]).

As pointed out in [CRS], the equality holds in the weighted anisotropic isoperimetric inequality in (4.1) whenever $\Sigma \cap E=\Sigma \cap B_{r}$, where $r$ is any positive number. That is, Wulff balls (centered at the origin) intersected with $\Sigma$ are always minimizers of the isoperimetric inequality (4.1). However, the uniquenes 2 of those minimizers (i.e., the characterization of the equality sign in (4.1)) in general is still not available in the literature (see Section 44). Whenever the uniqueness of those minimizers is available, it is not difficult to obtain the radial symmetry of $u$, as a corollary of Theorem 1.1

The uniqueness of the minimizers can be obtained in the unweighted (i.e., when $w \equiv 1$ ) anisotropic setting by adapting the ideas in FI] to the anisotropic setting (see Theorem 4.2). Notice that in the unweighted case we have $D=N$ (in Theorem 1.1), and (1.5) reads as follows:

$$
\begin{cases}-\operatorname{div}\left\{H(\nabla u)^{p-1} \nabla_{\xi} H(\nabla u)\right\}=f(u) & \text { in } \Sigma \cap \Omega, \\ u \geqslant 0 & \text { in } \Sigma \cap \Omega, \\ u=0 & \text { on } \Gamma_{0}, \\ \left\langle\nabla_{\xi} H(\nabla u), \nu\right\rangle=0 & \text { on } \Gamma_{1} \backslash\{0\} .\end{cases}
$$

In this setting, the uniqueness of the minimizers of the isoperimetric inequality and Theorem 1.1 lead to

Theorem 1.4 (Symmetry in convex cones in the anisotropic unweighted setting). Let $\Omega$ be a Wulff ball in $\mathbb{R}^{N}$ centered at $0, N \geqslant 2$, and let $1<p<\infty$. Assume that $f \in L_{\mathrm{loc}}^{\infty}([0, \infty))$ is nonnegative. Let $u \in C^{1}\left((\Sigma \cap \Omega) \cup \Gamma_{0} \cup \Gamma_{1} \backslash\{0\}\right) \cap W^{1, \infty}(\Sigma \cap \Omega)$ be a solution of (1.18) in the weak sense. Assume that either

$$
p \geqslant N
$$

or

$$
p<N \text { and, for some nonincreasing function } \phi \geqslant 0 \text {, we have } \phi \leqslant f \leqslant \frac{N p}{N-p} \phi .
$$

Then, $u$ is a radially symmetric and radially nonincreasing function. Moreover, by setting $M$ as in (1.15), $u$ is radially strictly decreasing on $\{0<u<M\}$,

and

$$
\{0<u<M\} \quad \text { is a Wulff annulus or a punctured Wulff ball centered at } 0 \text { intersected with } \Sigma \text {. }
$$

We point out that conditions $\left(\underline{\left.\mathrm{a}^{\prime}\right)}\right.$ and $\left(\underline{\mathrm{b}^{\prime}}\right)$ in Theorem 1.4 correspond, respectively, to conditions (国) and (B) of Theorem 1.1 when $D=N$.

Also, as usual, when $\Sigma=\mathbb{R}^{N}$, we have $\Gamma_{1}=\varnothing$ and the last condition in (1.18) is trivially satisfied. In this case, (1.18) simply becomes:

$$
\begin{cases}-\operatorname{div}\left\{H(\nabla u)^{p-1} \nabla_{\xi} H(\nabla u)\right\}=f(u) & \text { in } \Omega, \\ u \geqslant 0 & \text { in } \Omega, \\ u=0 & \text { on } \partial \Omega,\end{cases}
$$

and Theorem 1.4 reads as follows.

Theorem 1.5 (Symmetry in balls in the anisotropic unweighted setting). Let $\Omega$ be a Wulff ball in $\mathbb{R}^{N}$, $N \geqslant 2$, and let $1<p<\infty$. Assume that $f \in L_{\mathrm{loc}}^{\infty}([0, \infty))$ is nonnegative. Let $u \in C^{1}(\bar{\Omega})$ be a solution of (1.19) in the weak sense. Assume that either (a') or (

Then, $u$ is a radially symmetric and radially nonincreasing function. Moreover,

$$
u \text { is radially strictly decreasing on }\left\{0<u<\max _{\bar{\Omega}} u\right\} \text {, }
$$

and

$$
\left\{0<u<\max _{\bar{\Omega}} u\right\} \quad \text { is a Wulff annulus or a punctured Wulff ball. }
$$

\footnotetext{
${ }^{2}$ Whenever a line of direction $a \in \mathbb{R}^{N}$ is contained in $\Sigma$, uniqueness will be up to translations in direction $a$ (see Section 4).
} 
For completeness, in light of Remark 1.2, we point out that the regularity assumption on the solution $u$ taken in Theorem 1.5 can be relaxed by assuming only that the solution is bounded.

The uniqueness of the minimizers of the isoperimetric inequality is also available in the weighted isotropic setting (i.e., when $H$ is the Euclidean norm). In this case, (1.5) reads as follows

$$
\begin{cases}-\operatorname{div}\left\{w(x)|\nabla u|^{p-2} \nabla u\right\}=f(u) w(x) & \text { in } \Sigma \cap \Omega, \\ u \geqslant 0 & \text { in } \Sigma \cap \Omega, \\ u=0 & \text { on } \Gamma_{0}, \\ \langle\nabla u, \nu\rangle=0 & \text { on } \Gamma_{1} \backslash\{0\} .\end{cases}
$$

In this case, the uniqueness of the minimizers of the isoperimetric inequality and Theorem 1.1 lead $\sqrt{3}$ to

Theorem 1.6 (Symmetry in convex cones in the isotropic weighted setting). Let $\Omega$ be a (Euclidean) ball in $\mathbb{R}^{N}$ centered at $0, N \geqslant 2$, and let $1<p<\infty$. Assume that $f \in L_{\mathrm{loc}}^{\infty}([0, \infty))$ is nonnegative and the weight $w$ satisfies (1.7), (1.8), and (1.9). Let $u \in C^{1}\left((\Sigma \cap \Omega) \cup \Gamma_{0} \cup \Gamma_{1} \backslash\{0\}\right) \cap W^{1, \infty}(\Sigma \cap \Omega)$ be a solution of (1.20) in the weak sense.

Assume that either (国) or (b) of Theorem 1.1 holds true.

Then, $u$ is a radially symmetric and radially nonincreasing function. Moreover, by setting $M$ as in (1.15), $u$ is radially strictly decreasing on $\{0<u<M\}$,

and

$$
\{0<u<M\} \quad \text { is a annulus or a punctured ball centered at } 0 \text { intersected with } \Sigma .
$$

We point out that Theorem 1.5 provides a generalization to the anisotropic setting of the celebrated result contained in [GNN] and such a generalization is new even for $p=2$ whenever $N>2$. We recall that GNN] exploits the moving planes method, which seems not to be helpful for anisotropic problems. Instead, the method via integral (in)equalities used here (and based on $[\mathrm{L}$ ) turns out to be effective in this setting.

When $\Sigma \subsetneq \mathbb{R}^{N}$, the results presented here are new even in the isotropic and unweighted setting (i.e., when $H$ is the Euclidean norm and $w \equiv 1$ ) whenever $2 \neq p \neq N$. In the case $p=2$ and $\Sigma \subsetneq \mathbb{R}^{N}, N>2$, the symmetry result was previously obtained only relying on the moving planes method (and in the unweighted isotropic setting) in $[\mathrm{BP}$ : even in this special case, the present paper provides a new approach to the problem via integral (in)equalities (based on $[\mathrm{L}$ ) which complements $\mathrm{BP}$.

Also, the results obtained here in the isotropic and weighted setting (i.e., with $w \not \equiv 1$ ) are new for any $p$.

Theorems 1.4, 1.5 and 1.6 can be rephrased in terms of the variational formulation of (1.5), as follows. We restrict this formulation to the case when $f$ is a continuous nonlinearity to avoid problems with the differentiability of the functional.

Theorem 1.7 (Variational formulation of Theorems 1.4, 1.5 and 1.6). Let $\Omega$ be a Wulff ball in $\mathbb{R}^{N}$ centered at $0, N \geqslant 2$, and let $1<p<\infty$. Assume that $f$ is a continuous nonnegative function defined on $[0, \infty)$. Let

$$
u \in W_{\Gamma_{0}}^{1, \infty}(\Sigma \cap \Omega):=\left\{v \in W^{1, \infty}(\Sigma \cap \Omega): v=0 \text { on } \Gamma_{0}\right\}
$$

be a nonnegative critical point of the functional

$$
\int_{\Sigma \cap \Omega}\left\{\frac{H^{p}(\nabla u)}{p}-F(u)\right\} w(x) d \mathcal{H}^{N}, \quad \text { where } F(s):=\int_{0}^{s} f(\tau) d \tau .
$$

Assume that $u \in C^{1}\left((\Sigma \cap \Omega) \cup \Gamma_{1} \backslash\{0\}\right)$ and that, either

$$
\begin{aligned}
& H \text { is any uniformly elliptic norm of class } C^{2}\left(\mathbb{R}^{N} \backslash\{0\}\right), w \equiv 1 \text {, } \\
& \text { and either (at or (bolds true, }
\end{aligned}
$$

or

$H$ is the euclidean norm, $w$ is any weight satisfying (1.7), (1.8) and (1.9),

and either (a) or (b) of Theorem 1.1 holds true.

Then, $u$ is a radially symmetric and radially nonincreasing function. Moreover, by setting $M$ as in (1.15), $u$ is radially strictly decreasing on the set $\{0<u<M\}$, which is a Wulff annulus or a punctured Wulff ball centered at 0 intersected with $\Sigma$.

\footnotetext{
${ }^{3}$ The recent examples in [I] for isoperimetric minimizers that are half-balls instead of full balls may also yield interesting statements in terms of lack of symmetry of solutions to be compared to Theorem 1.6 here.
} 
We conclude this introduction with a comment on the proofs of Theorems 1.4, 1.5 and 1.6. As already mentioned, those theorems are obtained by putting together Theorem [1.1] and the characterization of the equality sign in the isoperimetric inequality (4.1). When $\Sigma$ is a convex cone containing no lines, then the characterization of the minimizers of the isoperimetric inequality forces the superlevel sets to be Wulff balls all centered at 0 (which is the vertex of $\Sigma$ ) intersected with $\Sigma$. This immediately gives the desired symmetry results. When $\Sigma$ contains lines instead, the characterization of minimizers still informs us that all the superlevel sets are Wulff balls intersected with $\Sigma$, but a further step is needed in order to prove that all of those Wulff balls are centered at 0. This step is accomplished in Lemma 5.2

The rest of this paper is organized as follows. Section 2 recalls the basics of the Finsler framework, placing the known results into a setting convenient for the applications that we have in mind.

Other useful auxiliary results are collected in Section [3] which contains a suitable maximum principle in cones, a Stampacchia-type result, some geometric differential inequalities and a Pohozaev-type identity.

In Section 4 we deal with some isoperimetric inequalities, leveraging on the existing literature CGPRS, CR, CRS, FI, FM, FMP and adapting all the previous results to the case under consideration in this paper.

The proofs of the main results in Theorems [1.1, 1.4, 1.5] and 1.6 are then contained in Section [5.

\section{Preliminaries And Setting}

Here we recall some basic facts on the Finsler framework.

Given the norm $H$ satisfying (1.1), (1.2) and (1.3) and the dual norm $H_{0}$ defined in (1.10), we observe that we can reconstruct $H$ in terms of $H_{0}$ as

$$
H(\xi)=\sup _{x \neq 0} \frac{\langle x, \xi\rangle}{H_{0}(x)} \quad \text { for } \quad \xi \in \mathbb{R}^{N}
$$

Consequently, it holds that

$$
|\langle\xi, \eta\rangle| \leqslant H(\xi) H_{0}(\eta), \quad \text { for any } \xi, \eta \in \mathbb{R}^{N} \text {. }
$$

The two convex sets $B^{H_{0}}$ and $B^{H}$, defined in (1.11) and (1.4) respectively, are both centrally symmetric and they are polar of each other. We denote by $B_{r}^{H_{0}}(x)$ the ball centered at the point $x$ with radius $r$ in the norm $H_{0}$, i.e.

Analogously, we define

$$
B_{r}^{H_{0}}(x):=\left\{y \in \mathbb{R}^{N}: H_{0}(x-y)<r\right\}
$$

$$
B_{r}^{H}(x):=\left\{\xi \in \mathbb{R}^{N}: H(x-\xi)<r\right\} .
$$

The sets $B_{r}^{H_{0}}(x)$ are named Wulff balls of $H$ centered at $x$ with radius $r$, and they are homothetic copies of the unitary Wulff ball $B^{H_{0}}$. We call Wulff shapes of $H$ centered at $x$ with radius $r$ the boundaries $\partial B_{r}^{H_{0}}(x)$ of $B_{r}^{H_{0}}(x)$, i.e.,

$$
\partial B_{r}^{H_{0}}(x):=\left\{y \in \mathbb{R}^{N}: H_{0}(x-y)=r\right\} .
$$

In what follows, to ease notation, we will omit the exponent $H_{0}$, that is, the Wulff ball (of $H$ ) $B_{r}^{H_{0}}(x)$ centered at $x$ of radius $r$ will be denoted just by $B_{r}(x)$.

Furthermore, from the homogeneity property of $H$ in (1.3) we have

$$
\left\langle\nabla_{\xi} H(\xi), \xi\right\rangle=H(\xi), \quad \text { for any } \xi \in \mathbb{R}^{N},
$$

where the left-hand side is taken to be 0 when $\xi=0$.

Given a measurable set $E \subset \mathbb{R}^{N}$, the anisotropic perimeter of $E$ in $\Sigma$ is defined by

$$
P_{H}(E ; \Sigma):=\sup \left\{\int_{E} \operatorname{div}(\sigma) d x: \sigma \in C_{c}^{1}\left(\Sigma ; \mathbb{R}^{N}\right), H_{0}(\sigma) \leqslant 1\right\} .
$$

When $H$ is the Euclidean norm, we recover the usual Euclidean perimeter of $E$ in $\Sigma$

Notice that

$$
P(E ; \Sigma):=\sup \left\{\int_{E} \operatorname{div}(\sigma) d x: \sigma \in C_{c}^{1}\left(\Sigma ; \mathbb{R}^{N}\right),|\sigma| \leqslant 1\right\} .
$$

$$
P_{H}(E ; \Sigma) \text { is finite if and only if } P(E ; \Sigma) \text { is finite. }
$$

Indeed, all norms in $\mathbb{R}^{N}$ are equivalent. Thus, there exist two positive constants $k_{1} \leqslant k_{2}$ such that $H$ satisfies

$$
k_{1}|\xi| \leqslant H(\xi) \leqslant k_{2}|\xi| \quad \text { for any } \xi \in \mathbb{R}^{N} .
$$

This and the homogeneity of $H$ give that

$$
\frac{1}{k_{2}}|\xi| \leqslant H_{0}(\xi) \leqslant \frac{1}{k_{1}}|\xi| \quad \text { for any } \xi \in \mathbb{R}^{N},
$$


and hence that

$$
k_{1} P(E ; \Sigma) \leqslant P_{H}(E ; \Sigma) \leqslant k_{2} P(E ; \Sigma)
$$

which gives (2.4).

If $E$ is a set of finite perimeter, then we can write that

$$
P(E ; \Sigma)=\mathcal{H}^{N-1}\left(\Sigma \cap \partial^{*} E\right)
$$

and that

$$
P_{H}(E ; \Sigma)=\int_{\Sigma \cap \partial^{*} E} H(\nu) d \mathcal{H}^{N-1},
$$

where $\partial^{*} E$ is the reduced (or measure theoretic) boundary of $E$ on which an outer (measure theoretical) unit normal vector $\nu$ is defined (see Gi and, for the anisotropic setting, AB ).

Recalling the definition of the weighted anisotropic perimeter in (1.12) and of the weighted volume of a set in (1.13), we notice that, if $w$ is positively homogeneous of degree $\lambda \geqslant 0$ and $D$ is the quantity defined in (1.14), the following relation holds true

$$
P_{w, H}(B ; \Sigma)=D w(\Sigma \cap B),
$$

where $B$ is the unitary Wulff ball, see [CRS, Formula (1.14)].

For more details on $P_{w, H}(E ; \Sigma)$ and for more general definitions, we refer the reader to $\mathrm{BBF}$ and $\mathrm{CRS}$ (see also CGPRS).

\section{Additonal ingredients FOR the PROOF OF the MAIN RESUlts}

We collect here some other auxiliary results that turn out to be handy for the proof of the main theorems, such as a maximum principle in cones, a Stampacchia-type result, some geometric differential inequalities and a Pohozaev-type identity.

We start by proving the following maximum principle in cones.

Lemma 3.1 (Maximum principle in cones). Let $u$ be a (weak) solution of

$$
\begin{cases}-\operatorname{div}\left\{w(x) H(\nabla u)^{p-1} \nabla_{\xi} H(\nabla u)\right\}=\widetilde{f}(x) w(x) & \text { in } \Sigma \cap \Omega, \\ u=0 & \text { on } \Gamma_{0}, \\ \left\langle\nabla_{\xi} H(\nabla u), \nu\right\rangle=0 & \text { on } \Gamma_{1} \backslash\{0\},\end{cases}
$$

where the weight $w$ is continuous in $\bar{\Sigma}$ and positive and locally Lipschitz in $\Sigma$. If $\widetilde{f} \in L^{\infty}(\Sigma \cap \Omega)$ is nonnegative, then $u$ is nonnegative. In particular, if $\widetilde{f} \equiv 0$, then $u \equiv 0$.

Proof. We set $u^{-}:=\max \{0,-u\}$ and we compute

$$
\begin{aligned}
0 \leqslant \int_{\Sigma \cap \Omega} H^{p}\left(\nabla u^{-}\right) w(x) d \mathcal{H}^{N} & =\int_{\{u<0\}} H^{p}(\nabla u) w(x) d \mathcal{H}^{N} \\
& =\int_{\{u<0\}} H(\nabla u)^{p-1}\left\langle\nabla_{\xi} H(\nabla u), \nabla u\right\rangle w(x) d \mathcal{H}^{N} \\
& =\int_{\{u<0\}} u \tilde{f} w(x) d \mathcal{H}^{N} \leqslant 0,
\end{aligned}
$$

where in the second equality we used (2.3) and in the third equality we used the boundary value problem. Thus, $\nabla u^{-}=0$ a.e. in $\Sigma \cap \Omega$. Moreover, by Poincaré inequality (which holds true since $u^{-}=0$ on $\Gamma_{0}$ and $\left.\mathcal{H}^{N-1}\left(\Gamma_{0}\right)>0\right)$ we find that $u^{-}=0$, and hence $u \geqslant 0$ a.e. in $\Sigma \cap \Omega$.

In what follows, we will exploit the following result regarding the singular set of solutions to the anisotropic weighted equation considered in the present paper. In the isotropic unweighted setting, the result is due to Lou (see [Lo, Theorem 1.1 and Corollary 1.1]). We mention that the results in [Lo] hold true for more general nonlinearities, namely $f \in L^{q}(\Omega)$ with $q>p / N$ and $q \geqslant 2$.

Lemma 3.2. Let $E \subset \mathbb{R}^{N}$ be a bounded domain. Let $f \in L_{\mathrm{loc}}^{\infty}(\mathbb{R})$ and $w \in C(\bar{E})$, with $w$ positive and locally Lipschitz in $E$. Let $u$ be a bounded weak solution of

$$
-\operatorname{div}\left\{w(x) H(\nabla u)^{p-1} \nabla_{\xi} H(\nabla u)\right\}=f(u) w(x) \text { in } E,
$$

that is, $u \in W^{1, p}(E) \cap L^{\infty}(E)$ and

$$
\int_{E} H(\nabla u)^{p-1}\left\langle\nabla_{\xi} H(\nabla u), \nabla \phi\right\rangle w(x) d \mathcal{H}^{N}=\int_{E} f \phi w(x) d \mathcal{H}^{N} \quad \text { for any } \phi \in C_{c}^{\infty}(E) .
$$

\footnotetext{
${ }^{4}$ We stress that the right-hand side of (2.7) means " $D$ multiplied by $w(\Sigma \cap B)$ " (recall also the notation in (1.13)); in particular, no confusion should arise with the derivative of $w$.
} 
Then, $f=0$ a.e. in $\{x \in E: \nabla u(x)=0\}$.

We remark that we are not making any assumptions on the regularity of $E$ in Lemma 3.2, As a matter of fact, in what follows we are going to apply this result with $E:=\Sigma \cap \Omega$.

Proof of Lemma 3.2. We start by proving that

$$
a(\nabla u):=H(\nabla u)^{p-1} \nabla_{\xi} H(\nabla u) \in W_{\mathrm{loc}}^{1,2}(E) .
$$

To prove this, we first observe that

$$
\text { if } \tilde{f} \in L_{\mathrm{loc}}^{\infty}(E) \text {, then a solution of }-\operatorname{div}(a(\nabla u))=\tilde{f} \text { in } E \text { satisfies (3.3). }
$$

Indeed, when $H$ is the Euclidean norm, this result can be found in [Lo, Lemma 2.1]. The anisotropic version of this result (for uniformly elliptic norms $H \in C^{2}\left(\mathbb{R}^{N} \backslash\{0\}\right)$ ) will appear in ACF].

Now, in order to prove (3.3), we notice that (3.1) can be rewritten as

$$
-\operatorname{div}\left\{H(\nabla u)^{p-1} \nabla_{\xi} H(\nabla u)\right\}=f(u)+H(\nabla u)^{p-1}\left\langle\frac{\nabla w}{w}, \nabla_{\xi} H(\nabla u)\right\rangle \text { in } E .
$$

Now we set

$$
\widetilde{f}(x):=f(u(x))+H(\nabla u(x))^{p-1}\left\langle\frac{\nabla w(x)}{w(x)}, \nabla_{\xi} H(\nabla u(x))\right\rangle
$$

and we observe that, in light of our assumptions on $f$ and $w$, and the interior $C^{1, \gamma}$-regularity of $u$ (which holds true by [To]), $\widetilde{f} \in L_{\text {loc }}^{\infty}(E)$. Here, we used that $u$ is bounded in order to apply [To]. We also used that $\frac{H(\xi)^{p}}{p} \in C^{1}\left(\mathbb{R}^{N}\right)$ (see e.g., [CFV, Lemma 2.2]). Thus, (3.3) follows from (3.4).

Now, for any $\varphi \in C_{c}^{\infty}(E)$ we set

$$
\phi(x):=\frac{|a(\nabla u(x))|}{\varepsilon+|a(\nabla u(x))|} \varphi(x)
$$

and we notice that $\phi \in W_{0}^{1,2}(E)$. By using $\phi$ as a test function in (3.2), we obtain that

$$
\begin{aligned}
\int_{E} \frac{|a(\nabla u)|}{\varepsilon+|a(\nabla u)|} \varphi(x) f w(x) d \mathcal{H}^{N}= & \int_{E} \frac{|a(\nabla u)|}{\varepsilon+|a(\nabla u)|}\langle a(\nabla u), \nabla \varphi\rangle w(x) d \mathcal{H}^{N} \\
& +\varepsilon \int_{E} \frac{\langle a(\nabla u), \nabla(|a(\nabla u)|)\rangle}{(\varepsilon+|a(\nabla u)|)^{2}} \varphi(x) w(x) d \mathcal{H}^{N} .
\end{aligned}
$$

By the Cauchy-Schwartz inequality we have that

$$
\varepsilon \frac{\langle a(\nabla u), \nabla(|a(\nabla u)|)\rangle}{(\varepsilon+|a(\nabla u)|)^{2}} \leqslant|\nabla(|a(\nabla u)|)|,
$$

and the last term is in $L^{1}(E)$ (actually in $L^{2}(E)$ ) and does not depend on $\varepsilon$. Notice also that

$$
\int_{E \backslash\{\nabla u=0\}} \frac{|a(\nabla u)|}{\varepsilon+|a(\nabla u)|} \varphi(x) f d \mathcal{H}^{N}=\int_{E} \frac{|a(\nabla u)|}{\varepsilon+|a(\nabla u)|} \varphi(x) f d \mathcal{H}^{N} .
$$

Thus, by letting $\varepsilon \rightarrow 0^{+}$, (3.5), (3.6) and Lebesgue's dominated convergence theorem give that

$$
\int_{E \backslash\{\nabla u=0\}} \varphi(x) f w(x) d \mathcal{H}^{N}=\int_{E}\langle a(\nabla u), \nabla \varphi\rangle w(x) d \mathcal{H}^{N},
$$

for any $\varphi \in C_{c}^{\infty}(E)$. Hence, this and (3.2) lead to

$$
\int_{E} \varphi(x) f w(x) d \mathcal{H}^{N}=\int_{E \backslash\{\nabla u=0\}} \varphi(x) f w(x) d \mathcal{H}^{N} \quad \text { for any } \varphi \in C_{c}^{\infty}(E),
$$

and the desired result follows.

In what follows, for $t \in(-\infty, M]$ (where $M$ is that defined in (1.15)), we consider the sets

$$
u^{-1}(t):=\{u=t\}:=\{x \in \Sigma \cap \Omega: u(x)=t\} \quad \text { and } \quad\{u>t\}:=\{x \in \Sigma \cap \Omega: u(x)>t\},
$$

which are contained in $\Sigma \cap \Omega$ by definition.

Also, we consider the distribution-type functions:

$$
I(t):=\int_{\{u>t\}} f(u) w(x) d \mathcal{H}^{N}, \quad \mu(t):=w(\{u>t\}),
$$

and

$$
K:=I^{\alpha} \mu^{\beta},
$$

for any $\alpha, \beta \in \mathbb{R}$. 
In the sequel, we will specify our choice of parameters $\alpha$ and $\beta$ as follows

$$
\alpha:=p^{\prime}=\frac{p}{p-1} \quad \text { and } \quad \beta:=\frac{p-D}{D(p-1)},
$$

where $D$ is the quantity defined in (1.14).

We notice that, when $w \equiv 1$ (and hence $D=N$ ), these distribution-type functions are precisely those used

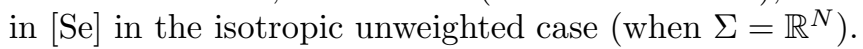

Lemma 3.3. Let $\alpha, \beta$ be arbitrary real numbers and $\Omega \subset \mathbb{R}^{N}$ be a bounded smooth domain. Assume that $u \in C^{0,1}(\overline{\Sigma \cap \Omega}) \cap C^{1}(\Sigma \cap \Omega)$ is nonnegative, $u=0$ on $\Gamma_{0}$, and let $w$ be as in Lemma 3.1. Let $f \in L_{\mathrm{loc}}^{\infty}([0, \infty))$ be nonnegative and let $I, \mu, K$ be those defined in (3.7) and (3.8). Then:

(i) The functions $I, \mu$ and $K$ are a.e. differentiable and

$$
-K^{\prime}(t)=\left[\alpha I(t)^{\alpha-1} \mu(t)^{\beta} f(t)+\beta I(t)^{\alpha} \mu(t)^{\beta-1}\right]\left(-\mu^{\prime}(t)\right) \quad \text { for a.e. } t .
$$

(ii) For a.e. $t \in(0, M)$, it holds that

$$
-\mu^{\prime}(t) \geqslant \int_{\{u=t\}} \frac{w}{|\nabla u|} d \mathcal{H}^{N-1} .
$$

Assume furthermore that $u$ is a weak solution of (1.5). Then:

(iii) If hypothesis (B) in Theorem 1.1 holds, then $I, \mu$ and $K$ are absolutely continuous functions for $t<M$.

(iv) Suppose in addition that $\alpha$ and $\beta$ are as in (3.9). Under the assumptions (回) and (b) in Theorem 1.1, $K(t)$ defined in (3.8) is nonincreasing for $t \in(0, M)$.

Proof. We take inspiration from the arguments used in [Se, Lemma 4] in the isotropic unweighted case $\Sigma=$ $\mathbb{R}^{N}$. More details and related questions can be found, e.g., in $\mathrm{BZ}$, Tal, $\mathrm{Fe}$.

(i) Since $I$ and $\mu$ are nonincreasing (by definition), they are a.e. differentiable. Also, they define nonpositive Lebesgue-Stieltjes measures $d I$ and $d \mu$ on $(0, M)$. By definition of Lebesgue integral, we find that

$$
I(t)=\int_{\{u>t\}} f(u) w(x) d \mathcal{H}^{N}=-\int_{t}^{M^{+}} f(\tau) d \mu(\tau),
$$

and hence $d I=f d \mu$. Thus, $I^{\prime}(t)=f(t) \mu^{\prime}(t)$ for $d \mu$-a.e. $t$. Now, since $w>0$ in $\Sigma$ and $|\nabla u|$ is bounded in $\{u \geqslant t\}, \mu$ is strictly decreasing. As a consequence, we have that the Lebesgue measure on $(0, M)$ is absolutely continuous with respect to $d \mu$. Thus, we get that

$$
I^{\prime}(t)=f(t) \mu^{\prime}(t) \text { for a.e. } t
$$

and (3.10) easily follows. In all the paper, unless otherwise indicated, a.e. is always with respect to the Lebesgue measure.

(ii) We define

$$
\mu_{0}(t):=w(\{u>t,|\nabla u|>0\}) .
$$

Let $\varepsilon>0$ and we use coarea formula to compute

$$
\begin{aligned}
w(\{u>t,|\nabla u|>\varepsilon\}) & =\int_{\Sigma \cap \Omega}|\nabla u| \frac{\chi_{\{u>t,|\nabla u|>\varepsilon\}} w(x)}{|\nabla u|} d \mathcal{H}^{N} \\
& =\int_{t}^{M} \int_{u^{-1}(\tau)} \frac{\chi_{\{u>t,|\nabla u|>\varepsilon\}} w(x)}{|\nabla u|} d \mathcal{H}^{N-1} d \tau \\
& =\int_{t}^{M} \int_{u^{-1}(\tau) \cap\{|\nabla u|>\varepsilon\}} \frac{w(x)}{|\nabla u|} d \mathcal{H}^{N-1} d \tau .
\end{aligned}
$$

By monotone convergence, letting $\varepsilon \rightarrow 0$, we find that (3.12) still holds true for $\varepsilon=0$ (and arbitrary $t$ ). Thus, $\mu_{0}(t)$ is absolutely continuous and

$$
-\mu_{0}^{\prime}(t)=\int_{u^{-1}(t) \cap\{|\nabla u|>0\}} \frac{w(x)}{|\nabla u|} d \mathcal{H}^{N-1} d \tau, \quad \text { for a.e. } t \in(0, M) .
$$

Again by using coarea formula we also find

and hence

$$
0=\int_{\Sigma \cap \Omega}|\nabla u| \chi_{\{u>t,|\nabla u|=0\}} d \mathcal{H}^{N}=\int_{t}^{M} \mathcal{H}^{N-1}\left(u^{-1}(\tau) \cap\{|\nabla u|=0\}\right) d \tau,
$$

$$
\mathcal{H}^{N-1}\left(u^{-1}(t) \cap\{|\nabla u|=0\}\right)=0 \text { for a.e. } t \text {. }
$$


Thus, we can replace (3.13) with

$$
-\mu_{0}^{\prime}(t)=\int_{u^{-1}(t)} \frac{w(x)}{|\nabla u|} d \mathcal{H}^{N-1} d \tau, \quad \text { for a.e. } t \in(0, M) .
$$

Also, for a.e. $t \in(0, M)$ (where both $\mu^{\prime}(t)$ and $\mu_{0}^{\prime}(t)$ exist) we have that

$$
-\mu^{\prime}(t)=\lim _{s \rightarrow t^{+}} \frac{w(s \geqslant u>t)}{s-t} \geqslant \lim _{s \rightarrow t^{+}} \frac{w(s \geqslant u>t,|\nabla u|>0)}{s-t}=-\mu_{0}^{\prime}(t) .
$$

Inequality (3.11) now follows from the latter equation and 3.15). We notice additionally that the equality sign holds for a.e. $t$ if $\{|\nabla u|=0\}$ has zero $\mathcal{H}^{N}$-measure.

(iii) If $p<D$ and $\phi \leqslant f \leqslant \frac{D p}{D-p} \phi$ for some nonincreasing function $\phi \geqslant 0$, then a solution of (1.5) satisfies

$$
-\operatorname{div}\left\{H(\nabla u)^{p-1} \nabla_{\xi} H(\nabla u)\right\}=0 \text { in }\left\{u \geqslant t_{0}\right\},
$$

where $t_{0} \in[0, \infty]$ satisfies that $\phi(t)>0$ for $t<0$ and $\phi(t) \equiv 0$ for $t>t_{0}$. Hence, by Lemma $\underline{3.1}$, if $t_{0}<+\infty$, we will have that $u \equiv t_{0}=M$ in $\left\{u \geqslant t_{0}\right\}$. Therefore, for every $t<M$ it holds that

$$
-\operatorname{div}\left\{H(\nabla u)^{p-1} \nabla_{\xi} H(\nabla u)\right\}=f(u) \geqslant \phi(u) \geqslant \phi(t)>0 \quad \text { in }\{0 \leqslant u<t\} .
$$

By using Lemma 3.2 (with $E:=\Sigma \cap \Omega$ ), we find that $f(u)$ vanishes a.e. in the set $\{|\nabla u|=0\} \cap\{0 \leqslant u<t\}$. Since $f(u) \geqslant \phi(u) \geqslant \phi(t)>0$ in $\{0 \leqslant u<t\}$, this is only possible if the singular set $\{|\nabla u|=0\} \cap\{0 \leqslant u<t\}$ has zero measure. Therefore, we have

$$
\mu(t)=\mu_{0}(t)+w(\{u=M\}) \quad \text { for every } t<M .
$$

Since $\mu_{0}(t)$ is absolutely continuous (as noticed in item (ii)), $\mu$ is an absolutely continuous function for $t<M$. Thus, also $I$ and $K$ are absolutely continuous for $t<M$.

(iv) Under hypothesis (国) of Theorem 1.1, the claim is obvious because $\alpha$ and $\beta$ in (3.9) are $\geqslant 0$. On the other hand, under hypothesis (b) we have that item (iii) in the statement of Lemma 3.3 applies and hence $K$ is absolutely continuous. Moreover, by item (i) we deduce that

$$
-K^{\prime} \geqslant 0 \text { a.e. }
$$

and hence $K$ is nonincreasing also in this case. To prove (3.16), we notice that in light of item (i), $-K^{\prime}(t)$ has the same sign as $\alpha f(t)+\beta I(t) / \mu(t)$, since $I, \mu,-\mu^{\prime}$ are nonnegative by definition. Thus, since $\beta<0$, we need $I(t) / \mu(t)+(\alpha / \beta) f(t) \leqslant 0$ a.e. Observing that $I(t) / \mu(t)$ is the mean of $f(u)$ over the superlevel set $u>t$, we easily conclude that a sufficient condition for $I / \mu+(\alpha / \beta) f \leqslant 0$ is that $f(s) \leqslant-(\alpha / \beta) f(t)$, whenever $s>t$. And this is satisfied if $\phi \leqslant f \leqslant-(\alpha / \beta) \phi$ for some nonincreasing $\phi \geqslant 0$. Replacing $\alpha, \beta$ by their values in (3.9) we obtain the condition (b) in Theorem 1.1 since $-\alpha / \beta=p D /(D-p)$.

Lemma 3.4 (Pohozaev-type identity). Let $\Sigma$ be an open convex cone in $\mathbb{R}^{N}$ and let $\Omega \subset \mathbb{R}^{N}$ be a bounded smooth domain such that $\partial \Gamma_{0}=\partial \Gamma_{1}$. Let $u \in C^{1}\left((\Sigma \cap \Omega) \cup \Gamma_{0} \cup\left(\Gamma_{1} \backslash\{0\}\right)\right) \cap W^{1, \infty}(\Sigma \cap \Omega)$ be a solution of (1.5), with $f \in L_{\mathrm{loc}}^{\infty}([0, \infty))$ nonnegative and $w$ satisfying (1.7) and (1.9). Let $D$ be as in (1.14).

Then, the following identity holds:

$$
D \int_{\Sigma \cap \Omega} F(u) w(x) d \mathcal{H}^{N}+\frac{p-D}{p} \int_{\Sigma \cap \Omega} u f(u) w(x) d \mathcal{H}^{N}=\frac{1}{p^{\prime}} \int_{\Gamma_{0}} H(\nabla u)^{p}\langle x, \nu\rangle w(x) d \mathcal{H}^{N-1},
$$

where

$$
F(s):=\int_{0}^{s} f(\tau) d \tau
$$

Proof. Due to lack of regularity in our setting, in order to prove (3.17) we argue by approximation. We approximate $\Sigma \cap \Omega$ by Lipschitz domains $E_{\delta}$ obtained by chopping off a $\delta$-neighborhood of 0 (in the case $0 \in \Gamma_{1}$ ) and a $\delta$-tubular neighborhood of $\partial \Gamma_{0}$.

Step 1. We prove that for any $\eta \in C_{c}^{0,1}\left(E_{\delta}\right)$ we have that

$$
-\int_{E_{\delta}} \mathcal{R} \nabla \eta d \mathcal{H}^{N}=\int_{E_{\delta}} \eta(x) w(x)\left\{(N+\lambda) F(u)+\frac{p-(N+\lambda)}{p} u f(u)\right\} d \mathcal{H}^{N},
$$

where

$$
\begin{aligned}
\mathcal{R}:=w(x)\left\{x\left(F(u)-\frac{H(\nabla u)^{p}}{p}\right)\right. & +\langle x, \nabla u\rangle H(\nabla u)^{p-1} \nabla_{\xi} H(\nabla u) \\
& \left.+\left(\frac{(N+\lambda)-p}{p}\right) u H(\nabla u)^{p-1} \nabla_{\xi} H(\nabla u)\right\} .
\end{aligned}
$$

For any given $\eta \in C_{c}^{0,1}\left(E_{\delta}\right)$, we consider a smooth (say $C^{1, \gamma}$ ) open set $A \subset \subset E_{\delta}$ such that $\operatorname{supp}(\eta) \subset A$. 
Furthermore, by setting

we see that $u$ satisfies the equation

$$
\psi(t):=\frac{t^{p}}{p}, \quad \text { for } t \geqslant 0
$$

$$
-\operatorname{div}\left(w(x) \psi^{\prime}(H(\nabla u)) \nabla_{\xi} H(\nabla u)\right)=f(u) w(x) \quad \text { in } \Sigma \cap \Omega .
$$

For $t \geqslant 0$ and $\varepsilon \in(0,1)$, we let

$$
\psi_{\varepsilon}(t):=\psi\left(\sqrt{\varepsilon^{2}+t^{2}}\right)-\psi(\varepsilon) .
$$

We define $\Psi(t):=\psi^{\prime}(t) t$ and $\Psi_{\varepsilon}(t):=\psi_{\varepsilon}^{\prime}(t) t$. From a standard argument (see for instance [CFV], Lemma 4.2] or [BC, Lemma 3.2]) we have that

$$
\psi_{\varepsilon} \rightarrow \psi \quad \text { and } \quad \Psi_{\varepsilon} \rightarrow \Psi \text { uniformly on compact sets of }[0,+\infty) \text {. }
$$

Let $u_{\varepsilon}$ be solution of

$$
\left\{\begin{array}{l}
\operatorname{div}\left(w(x) \psi_{\varepsilon}^{\prime}\left(H\left(\nabla u_{\varepsilon}\right)\right) \nabla_{\xi} H\left(\nabla u_{\varepsilon}\right)\right)=f(u) w(x) \quad \text { in } A \\
u_{\varepsilon}=u \text { on } \partial A .
\end{array}\right.
$$

Standard regularity results give that $u_{\varepsilon} \in C^{1, \gamma}(\bar{A}) \cap W_{\mathrm{loc}}^{2,2}(A)$ and $u_{\varepsilon} \rightarrow u$ in $C^{1}(\bar{A})$, as $\varepsilon \rightarrow 0$. For example, one can obtain $u_{\varepsilon}$ by minimizing the functional

$$
\int_{A}\left\{\psi_{\varepsilon}(H(\nabla v))-f(u(x)) v\right\} w(x) d \mathcal{H}^{N}
$$

among functions $v \in W^{1, p}(A)$ with $v-u \in W_{0}^{1, p}(A)$, and then exploit arguments similar to those used in CFV, Proposition 4.3].

Notice that, since $A \subset \subset \Sigma$, we have that $w \in C^{0,1}(\bar{A})$ and $w>0$ on $\bar{A}$ and hence the results of regularity (up to the boundary) contained in $\mathrm{Li}$ can be used on $A$.

Now we set

$$
\begin{aligned}
\mathcal{R}_{\varepsilon}:=w(x)\left\{x\left(F(u)-\psi_{\varepsilon}\left(H\left(\nabla u_{\varepsilon}\right)\right)\right)\right. & +\left\langle x, \nabla u_{\varepsilon}\right\rangle \psi_{\varepsilon}^{\prime}\left(H\left(\nabla u_{\varepsilon}\right)\right) \nabla_{\xi} H\left(\nabla u_{\varepsilon}\right) \\
& \left.+\left(\frac{(N+\lambda)-p}{p}\right) u_{\varepsilon} \psi_{\varepsilon}^{\prime}\left(H\left(\nabla u_{\varepsilon}\right)\right) \nabla_{\xi} H\left(\nabla u_{\varepsilon}\right)\right\}
\end{aligned}
$$

and we exploit [PS, Proposition 1] and [PS, Remark at page 685] with

$$
\begin{aligned}
& \mathcal{F}:=\mathcal{F}_{\varepsilon}\left(x, \nabla u_{\varepsilon}\right):=\left\{\psi_{\varepsilon}\left(H\left(\nabla u_{\varepsilon}\right)\right)-F(u(x))\right\} w(x), \quad h:=x, \quad a:=\frac{(N+\lambda)-p}{p} \\
& \text { and } \quad \mathcal{G}(x):=-f(u(x)) w(x),
\end{aligned}
$$

equation (2.3), and the fact that $\langle x, \nabla w\rangle=\lambda w(x)$ in $\Sigma$ (which holds true in light of the $\lambda$-homogeneity of $w$ ), to see that

$$
\begin{aligned}
\operatorname{div}\left(\mathcal{R}_{\varepsilon}\right)=(N & +\lambda) F(u) w(x)+\frac{p-(N+\lambda)}{p} u_{\varepsilon} f(u) w(x) \\
& +(N+\lambda) w(x)\left\{\frac{\Psi_{\varepsilon}\left(H\left(\nabla u_{\varepsilon}\right)\right)}{p}-\psi_{\varepsilon}\left(H\left(\nabla u_{\varepsilon}\right)\right)\right\} \\
& +w(x)\left\{F^{\prime}(u)\langle x, \nabla u\rangle-f(u)\left\langle x, \nabla u_{\varepsilon}\right\rangle\right\}
\end{aligned}
$$

in the weak sense in $A$. By recalling that $\eta \in C_{c}^{0,1}\left(E_{\delta}\right)$, and that $\operatorname{supp}(\eta) \subset A$, we thus can write that

$$
\begin{aligned}
-\int_{E_{\delta}} \mathcal{R}_{\varepsilon} \nabla \eta d \mathcal{H}^{N}=\int_{E_{\delta}} & \eta(x) w(x)\left\{(N+\lambda) F(u)+\frac{p-(N+\lambda)}{p} u_{\delta}^{\varepsilon} f(u)\right\} d \mathcal{H}^{N} \\
& +(N+\lambda) \int_{E_{\delta}} \eta(x) w(x)\left\{\frac{\Psi_{\varepsilon}\left(H\left(\nabla u_{\varepsilon}\right)\right)}{p}-\psi_{\varepsilon}\left(H\left(\nabla u_{\varepsilon}\right)\right)\right\} d \mathcal{H}^{N} \\
& +\int_{E_{\delta}} \eta(x) w(x)\left\{F^{\prime}(u)\langle x, \nabla u\rangle-f(u)\left\langle x, \nabla u_{\varepsilon}\right\rangle\right\} d \mathcal{H}^{N} .
\end{aligned}
$$

We also note that

$$
\frac{\Psi(H(\nabla u))}{p}-\psi(H(\nabla u))=0
$$


Moreover, since $F^{\prime}(t)=f(t)$ for a.e. $t$, and hence $F^{\prime}(u(x))=f(u(x))$ for a.e. $x \in\{\nabla u \neq 0\}$, we thus have that

$$
\begin{gathered}
\int_{E_{\delta}} \eta(x) w(x) F^{\prime}(u)\langle x, \nabla u\rangle d \mathcal{H}^{N}=\int_{E_{\delta} \cap\{\nabla u \neq 0\}} \eta(x) w(x) f(u)\langle x, \nabla u\rangle d \mathcal{H}^{N} \\
=\int_{E_{\delta}} \eta(x) w(x) f(u)\langle x, \nabla u\rangle d \mathcal{H}^{N} .
\end{gathered}
$$

This implies that

$$
\int_{E_{\delta}} \eta(x) w(x)\langle x, \nabla u\rangle\left\{F^{\prime}(u)-f(u)\right\} d \mathcal{H}^{N}=0 .
$$

Hence, by taking the limit for $\varepsilon \rightarrow 0$ in (3.20), we obtain (3.18).

Step 2. For $k \geqslant 1$ we define $\phi_{k}: \mathbb{R} \rightarrow[0,1]$ as follows

$$
\phi_{k}(s):= \begin{cases}0 & \text { if } s \leqslant \frac{1}{k} \\ k s-1 & \text { if } \frac{1}{k}<s<\frac{2}{k} \\ 1 & \text { if } s \geqslant \frac{2}{k}\end{cases}
$$

Then we define $\eta_{k} \in C_{c}^{0,1}\left(E_{\delta}\right)$ as

$$
\eta_{k}(x):=\phi_{k}\left(\operatorname{dist}\left(x, \mathbb{R}^{N} \backslash E_{\delta}\right)\right)
$$

In this way we have that

$$
\eta_{k}(x) \rightarrow 1 \quad \text { for every } x \in E_{\delta}
$$

and that (see, e.g., DMS, Formula (21)], [CDLP, Section 7] and [AFP, Chapter 3])

$$
\lim _{k} \int_{E_{\delta}}\left\langle v, \nabla \eta_{k}\right\rangle d \mathcal{H}^{N}=-\int_{\partial E_{\delta}}\langle v, \nu\rangle d \mathcal{H}^{N-1}, \quad \text { for any } v \in C\left(\bar{E}_{\delta} ; \mathbb{R}^{N}\right) \text {. }
$$

Now we choose $\eta:=\eta_{k}$ in (3.18) and we take the limit as $k \rightarrow \infty$, using (3.21) (with $v=\mathcal{R}$ ) and taking into account that the boundary conditions in $(1.5)$ and the geometry of $\Sigma$ give that

where we have set

$$
\begin{gathered}
u=0 \quad \text { and } \quad F(u)=0 \quad \text { on } \Gamma_{0, \delta}, \\
\left\langle\nabla_{\xi} H(\nabla u), \nu\right\rangle=0 \quad \text { and } \quad\langle x, \nu\rangle=0 \quad \text { on } \Gamma_{1, \delta},
\end{gathered}
$$

$$
\Gamma_{0, \delta}:=\Gamma_{0} \cap \partial E_{\delta}, \quad \Gamma_{1, \delta}:=\Gamma_{1} \cap \partial E_{\delta} \quad \text { and } \quad \Gamma_{\delta}:=\partial E_{\delta} \backslash\left(\Gamma_{0, \delta} \cup \Gamma_{1, \delta}\right) .
$$

Thus, we obtain that

$$
\begin{aligned}
\int_{\Gamma_{0}, \delta} w(x) & \left\{\langle x, \nabla u\rangle H^{p-1}(\nabla u)\left\langle\nabla_{\xi} H(\nabla u), \nu\right\rangle-\langle x, \nu\rangle \frac{H(\nabla u)^{p}}{p}\right\} d \mathcal{H}^{N-1}+\int_{\Gamma_{\delta}}\langle\mathcal{R}, \nu\rangle d \mathcal{H}^{N-1} \\
& =\int_{E_{\delta}} w(x)\left\{(N+\lambda) F(u)-\frac{(N+\lambda)-p}{p} u f(u)\right\} d \mathcal{H}^{N} .
\end{aligned}
$$

By using that

$$
\nabla u=-|\nabla u| \nu \quad \text { on } \Gamma_{0, \delta}
$$

(which holds true in light of the boundary condition on $\Gamma_{0}$ in (1.5) ) and (2.3), the previous identity becomes

$$
\begin{aligned}
& \frac{1}{p^{\prime}} \int_{\Gamma_{0, \delta}} w(x) H(\nabla u)^{p}\langle x, \nu\rangle d \mathcal{H}^{N-1}+\int_{\Gamma_{\delta}}\langle\mathcal{R}, \nu\rangle d \mathcal{H}^{N-1} \\
= & \int_{E_{\delta}} w(x)\left\{(N+\lambda) F(u)-\frac{(N+\lambda)-p}{p} u f(u)\right\} d \mathcal{H}^{N},
\end{aligned}
$$

where $p^{\prime}=p /(p-1)$. Then we take the limit as $\delta \rightarrow 0$. Since $u \in W^{1, \infty}(\Sigma \cap \Omega)$ and $\mathcal{H}^{N-1}\left(\Gamma_{\delta}\right)$ tends to 0 as $\delta \rightarrow 0$, we have that the integral over $\Gamma_{\delta}$ tends to 0 . Recalling that $D=N+\lambda$, (3.17) follows. 


\section{ISOPERIMETRIC INEQUALITIES IN CONVEX CONES}

In this section, we revisit the existing literature on isoperimetric inequalities in convex cones and, providing several technical improvements to the previous results, we frame the isoperimetric theory into a convenient setting for our applications.

Theorem 4.1 (Weighted anisotropic isoperimetric inequality in cones, [CRS]). Let $\Sigma$ be an open convex cone in $\mathbb{R}^{N}$ with vertex at the origin and let $B$ be the unitary Wulff ball centered at the origin. Let $w$ satisfy (1.7), (1.8), and (1.9). Then, for each measurable set $E \subset \mathbb{R}^{N}$ with $w(\Sigma \cap E)<\infty$,

$$
\frac{P_{w, H}(E ; \Sigma)}{w(\Sigma \cap E)^{\frac{D-1}{D}}} \geqslant \frac{P_{w, H}(B ; \Sigma)}{w(\Sigma \cap B)^{\frac{D-1}{D}}},
$$

where $D=N+\lambda$, and $\lambda$ is that in (1.7).

Theorem 4.1 has been obtained in by Cabré-Ros-Oton-Serra in [CRS] in the more general setting in which $H$ is a gauge. Since we do not need such a generality here, we have stated it assuming that $H$ is a norm.

As mentioned in the introduction, Wulff balls centered at the origin intersected with $\Sigma$ are always minimizers of (4.1). Let us discuss about the uniqueness of those minimizers, or, in other words, about the characterization of the equality sign in (4.1).

In CRS the authors do not provide the characterization of the equality case, stating that such a characterization will be postponed in a future work. To the authors' knowledge such a characterization among general sets in the unweighted anisotropic setting of Theorem 4.1 is still not available in the literature.

We recall that the isoperimetric inequality (4.1) in the unweighted isotropic setting (i.e., when $w \equiv 1$ and $H$ is the Euclidean norm) is due to Lions-Pacella $[\mathrm{LP}$. In this setting, they also obtained the characterization of the equality case when $\Sigma \backslash\{0\}$ is assumed to be smooth.

Quantitative versions of inequality (4.1) in the unweighted isotropic setting (i.e., when $w \equiv 1$ and $H$ is the Euclidean norm) including the characterization of the equality case (in cones that are not necessarily smooth outside the origin) have been obtained by Figalli-Indrei in [FI]. In Theorem 4.2 below we show that their proof can be extended to the case of a general norm $H$.

A quantitative version of inequality (4.1) in the weighted isotropic setting (i.e., with $w$ as in Theorem 4.1 and $H$ the Euclidean norm) including the characterization of the equality case (in cones that are not necessarily smooth outside the origin) has been recently obtained by Cinti-Glaudo-Pratelli-Ros-Oton-Serra in CGPRS.

A different proof of Theorem 4.1 has been obtained by Milman-Rotem in $\mathrm{MR}$. There, the authors also provide a characterization under the additional assumption that $\Sigma \cap E$ is convex 5 . Due to the convexity restriction, we cannot apply their characterization in the present paper. In fact, in order to obtain Theorms 1.4, 1.5, 1.6 from Theorem 1.1, we need to use such a characterization on (almost all) the superlevel sets $\{u>t\}$, and we do not know a priori whether those sets are convex or not.

Theorem 4.2 (Characterization of minimizers in the unweighted anisotropic isoperimetric inequality in convex cones). Let $\Sigma$ be an open convex cone in $\mathbb{R}^{N}$ with vertex at 0 and let $B$ be the unitary Wulff ball centered at 0 . Then,

$$
\frac{P_{H}(E ; \Sigma)}{\mathcal{H}^{N}(\Sigma \cap E)^{\frac{N-1}{N}}} \geqslant \frac{P_{H}(B ; \Sigma)}{\mathcal{H}^{N}(\Sigma \cap B)^{\frac{N-1}{N}}},
$$

for every measurable set $E \subset \mathbb{R}^{N}$ with $\mathcal{H}^{N}(\Sigma \cap E)<\infty$.

Moreover, up to rotations, we write $\Sigma=\mathbb{R}^{k} \times \tilde{\Sigma}$, where $0 \leqslant k \leqslant N$ and $\tilde{\Sigma} \subset \mathbb{R}^{N-k}$ is an open convex cone containing no lines, and the equality sign holds in (4.2) if and only if $E$ is a Wulff ball of some radius $r>0$ centered at $x_{0} \in \mathbb{R}^{k} \times\left\{0_{\mathbb{R}^{N-k}}\right\}$.

As already mentioned, when $H$ is the Euclidean norm, the proof of Theorem 4.2 can be found in [FI]. There, the authors observe that the (isotropic) isoperimetric inequality in cones (namely formula (5.7) with $H$ being the Euclidean norm) can be obtained as an immediate corollary of the anisotropic isoperimetric inequality

$$
\frac{P_{K}(E)}{\mathcal{H}^{N}(E)^{\frac{N-1}{N}}} \geqslant N \mathcal{H}^{N}(K)^{\frac{1}{N}} .
$$

Here, $K$ is an open bounded convex set and, for a set $E$ of finite perimeter (i.e., $\left.P\left(E ; \mathbb{R}^{N}\right)<\infty\right)$,

$$
P_{K}(E):=\int_{\partial^{*} E}\left\|\nu_{E}(x)\right\|_{K_{0}} d \mathcal{H}^{N-1}
$$

\footnotetext{
${ }^{5}$ MR has been published in [Adv. Math.262, 2014, 867-908]. In the published version, the convexity assumption in the characterization of minimizers was missing. This has been later fixed in $\mathrm{MR}$ (see footnote 1 in $[\mathrm{MR}]$ ).
} 
where $\partial^{*} E$ is the reduced boundary of $E, \nu_{E}$ is the measure theoretic outer unit normal, and

$$
\|\nu\|_{K_{0}}:=\sup \{\langle\nu, z\rangle: z \in K\} .
$$

Inequality (4.3) and its characterization of the equality case are well known in the literature. We refer to Di, Scha, DP, Ta, [FM, BM, [MS, [FI] and references therein.

As noticed in [FI], the choice $K:=\Sigma \cap B$, being $B$ the unitary Euclidean ball centered at the origin allows to recover (4.2) when $H$ is the Euclidean norm. This also allows to obtain the desired characterization. We will show that their argument still applies when $H$ is any norm in $\mathbb{R}^{N}$.

Proof of Theorem 4.2. The strategy is to extend to a more general $H$ the arguments used in [FI, Theorem 2.2]. To this aim, we set

$$
K:=\Sigma \cap B, \quad \text { where } B \text { is the unitary Wulff ball. }
$$

Let now $E$ be a set contained in $\Sigma$ and with finite perimeter. We notice that, if $z \in K$, then $H_{0}(z) \leqslant 1$, and hence by (2.2),

$$
\left\|\nu_{E}\right\|_{K_{0}}:=\sup \left\{\left\langle\nu_{E}, z\right\rangle: z \in K\right\} \leqslant H\left(\nu_{E}\right)
$$

By definition of $\|\cdot\|_{K_{0}}$ it easily follows that $\left\|\nu_{\Sigma}\right\|_{K_{0}}=0$ for $\mathcal{H}^{N-1}$-a.e. $x \in \partial \Sigma \backslash\{0\}$, and hence also for $\mathcal{H}^{N-1}$-a.e. $x \in \partial \Sigma \cap \partial^{*} E$. Thus,

$$
\begin{aligned}
P_{K}(E) & =\int_{\partial^{*} E}\left\|\nu_{E}(x)\right\|_{K_{0}} d \mathcal{H}^{N-1} \\
& =\int_{\Sigma \cap \partial^{*} E}\left\|\nu_{E}(x)\right\|_{K_{0}} d \mathcal{H}^{N-1} \\
& \leqslant \int_{\Sigma \cap \partial^{*} E} H\left(\nu_{E}(x)\right) d \mathcal{H}^{N-1} \\
& =P_{H}(E ; \Sigma),
\end{aligned}
$$

where in the last identity we used (2.6). In light of the inequality

$$
P_{K}(E) \leqslant P_{H}(E ; \Sigma)
$$

and recalling (2.7) (with $w \equiv 1$ ), it is clear that (4.2) follows from (4.3).

Now assume that $E$ satisfies the equality in (4.2). In light of (4.4), $E$ will also satisfy the equality in (4.3). By rescaling, if necessary, we may assume $\mathcal{H}^{N}(E)=\mathcal{H}^{N}(K)$. Thus, we have that

$$
P(K ; \Sigma)=N \mathcal{H}^{N}(K)=P_{K}(E)=P_{H}(E ; \Sigma),
$$

where the first equality follows from (2.7) (with $w \equiv 1$ ).

By [FM] (see also [FMP, Theorem A.1]), we obtain that

$$
E=K+a \quad \text { with } a \in \bar{\Sigma} \text {. }
$$

Notice that, for any $v \in \bar{\Sigma}$ it holds that

$$
P_{H}(v+K ; \Sigma)=P_{H}(K ; \Sigma)+P_{H}\left(S_{v}\right),
$$

where

$$
S_{v}:=\left\{x \in \partial^{*} \Sigma \cap B:\left\langle\nu_{\Sigma}(x), v\right\rangle \neq 0\right\}=\left\{x \in \partial^{*} \Sigma \cap B:\left\langle\nu_{\Sigma}(x), v\right\rangle<0\right\} .
$$

By putting together (4.5), (4.6) and (4.7), we get that

$$
P(K ; \Sigma)=P_{H}(E ; \Sigma)=P_{H}(K ; \Sigma)+P_{H}\left(S_{a}\right),
$$

and hence $P_{H}\left(S_{a}\right)=0$. By recalling (2.5) we find that $\mathcal{H}^{N-1}\left(S_{a}\right)=0$, and hence that $\left\langle\nu_{\Sigma}(x), a\right\rangle=0$ for $\mathcal{H}^{N-1}$-a.e. $x \in \partial^{*} \Sigma$. As in [FI], this gives that the distributional derivative of $\chi_{\Sigma}$ is zero in the direction defined by $a$ and hence that

$$
\chi_{\Sigma}(x)=\chi_{\Sigma}(x+t a) \quad \text { for all } t \in \mathbb{R} .
$$

Since $\Sigma=\mathbb{R}^{k} \times \tilde{\Sigma}$, where $0 \leqslant k \leqslant n$ and $\tilde{\Sigma} \subset \mathbb{R}^{N-k}$ contains no lines, we find that $a \in \mathbb{R}^{k} \times\left\{0_{\mathbb{R}^{N-k}}\right\}$. This concludes the proof.

In the weighted isotropic setting, CGPRS, Proposition 1.2] provides the characterization of the equality case.

Theorem 4.3 (Characterization of minimizers in the weighted isotropic isoperimetric inequality in convex cones, [CGPRS]. Up to rotations, we can write $\Sigma=\mathbb{R}^{k} \times \tilde{\Sigma}$, where $0 \leqslant k \leqslant N$ and $\tilde{\Sigma} \subset \mathbb{R}^{N-k}$ is an open convex cone containing no lines. Assume that $H$ is the Euclidean norm. Then, the equality sign holds in (4.1) (with $H$ the Euclidean norm) if and only if $E$ is a (Euclidean) ball of some radius $r>0$ centered at $x_{0} \in \mathbb{R}^{k} \times\left\{0_{\mathbb{R}^{N-k}}\right\}$. 
We point out that CGPRS, Proposition 1.2] is stated for $0 \leqslant k<N$. However, notice that in the case $k=N$ (i.e., $\Sigma=\mathbb{R}^{N}$ ), (4.1) reduces to the unweighted anisotropic isoperimetric inequality in $\mathbb{R}^{N}$, whose characterization is well known in the literature (and of course is contained in Theorem 4.2). In fact, the assumptions on the weight $w$ in Theorem 4.1 force $w$ to be (a positive) constant whenever $\Sigma=\mathbb{R}^{N}$ (see, e.g., [CR. Remark 3.8 and Lemma 3.9] and [CRS).

For other results and more information on weighted isoperimetric inequalities, we refer to Frank Morgan's blog [Mo, Ch, CRS, CGPRS], and references therein.

Remark 4.4. In order to describe the results concerning the characterization of minimizers we used that, for any open convex cone $\Sigma \subseteq \mathbb{R}^{N}$, without loss of generality, up to rotations, we can write $\Sigma=\mathbb{R}^{k} \times \tilde{\Sigma}$, where $0 \leqslant k \leqslant N$ and $\tilde{\Sigma} \subset \mathbb{R}^{N-k}$ is an open convex cone containing no lines.

\section{Proof of Theorems [1.1, 1.4, 1.5] And 1.6}

In this section, we exploit the results of the previous sections to prove Theorems 1.1, 1.4, 1.5] and 1.6. To this end, the following Gauss-Green-type identity and Hölder-isopertimetric-type inequality will be useful.

Lemma 5.1. Suppose that the assumptions of Theorem 1.1 are satisfied. Let I be the function defined in (3.7). Then, we have the following:

(i) The Gauss-Green-type identity holds true, namely

$$
I(t)=\int_{\{u=t\}} H(\nabla u)^{p-1} H(\nu) w(x) d \mathcal{H}^{N-1} \quad \text { for a.e. } t \in(0, M) .
$$

(ii) The Hölder-isopertimetric-type inequality holds true, namely

$$
I(t)^{\frac{1}{p}}\left(\int_{\{u=t\}} \frac{w(x)}{|\nabla u|} d \mathcal{H}^{N-1}\right)^{\frac{p-1}{p}} \geqslant c \mu(t)^{\frac{D-1}{D}}, \quad \text { for a.e. } t \in(0, M),
$$

where

$$
c:=\frac{P_{w, H}(B ; \Sigma)}{w(\Sigma \cap B)^{\frac{D-1}{D}}}
$$

is the optimal constant in the weighted anisotropic isoperimetric inequality in cones (4.1), being $B$ the unitary Wulff ball centered at the origin.

Moreover, the equality sign holds in (5.2) if and only if $\{u>t\}$ is a minimizer of (4.1) and $H(\nabla u)$ is constant on $\{u=t\}$.

Proof. (i) Since the function $u$ is of bounded variation in $\Sigma \cap \Omega$, the coarea theorem for BV functions (see e.g. [EG, Theorem 1, Section 5.5]) guarantees that the sets $\{u>t\}$ have finite perimeter for a.e. $t$. For the measure theoretic boundary $\partial^{*}\{u>t\}$, we see that

$$
\{u=t\} \cap\{|\nabla u|>0\} \subset \Sigma \cap \partial^{*}(\{u>t\}) \subset\{u=t\} .
$$

By recalling (3.14), we conclude that

$$
\mathcal{H}^{N-1}(\{u=t\} \cap\{|\nabla u|>0\})=\mathcal{H}^{N-1}\left(\Sigma \cap \partial^{*}(\{u>t\})\right) \text { for a.e. } t,
$$

and the exterior measure theoretical normal vector $\nu$ of $\partial^{*}\{u>t\}$ satisfies, a.e. in the sense of the measure $\mathcal{H}^{N-1}$ :

$$
|\nabla u| \nu=-\nabla u \quad \mathcal{H}^{N-1}-\text { a.e. on } \Sigma \cap \partial^{*}(\{u>t\}) .
$$

Accordingly, by using (1.5) (we are using the differential equation together with the homogeneous boundary condition on $\Gamma_{1}$ ) and De Giorgi's structure theorem (see [Ma, Theorem 15.9] or [Gi]), we have, up to a standard approximation argument,

$$
I(t)=\int_{\{u>t\}} f(u) w(x) d \mathcal{H}^{N}=\int_{\Sigma \cap \partial^{*}\{u>t\}} H(\nabla u)^{p-1}\left\langle\nabla_{\xi} H(\nabla u), \nu\right\rangle w(x) d \mathcal{H}^{N-1}, \text { for a.e. } t \in(0, M)
$$

and hence, by using (2.3) and (5.5),

$$
I(t)=\int_{\Sigma \cap \partial^{*}\{u>t\}} H(\nabla u)^{p-1} H(\nu) w(x) d \mathcal{H}^{N-1}, \quad \text { for a.e. } t \in(0, M) .
$$

By (3.14) and (5.4), we have that

$$
\int_{\Sigma \cap \partial^{*}\{u>t\}} H(\nabla u)^{p-1} H(\nu) w(x) d \mathcal{H}^{N-1}=\int_{\{u=t\}} H(\nabla u)^{p-1} H(\nu) w(x) d \mathcal{H}^{N-1} \text {, for a.e. } t \in(0, M),
$$

and the conclusion follows. 
(ii) By the isoperimetric inequality (4.1), we have

$$
P_{w, H}(\{u>t\} ; \Sigma) \geqslant c w(\{u>t\})^{\frac{D-1}{D}}=c \mu(t)^{\frac{D-1}{D}}, \quad \text { for a.e. } t \in(0, M),
$$

where $c$ is the optimal (weighted anisotropic) isoperimetric constant defined in (5.3).

Now, by the homogeneity of $H$ and (5.5),

$$
H(\nu) H(\nabla u)^{p-1}=H(\nu)^{p}|\nabla u|^{p-1} .
$$

By using this and (5.1), we compute that

$$
\begin{aligned}
I(t)^{\frac{1}{p}}\left(\int_{\{u=t\}} \frac{w(x)}{|\nabla u|} d \mathcal{H}^{N-1}\right)^{\frac{p-1}{p}} & =\left(\int_{\{u=t\}} H(\nu)^{p}|\nabla u|^{p-1} w(x) d \mathcal{H}^{N-1}\right)^{\frac{1}{p}}\left(\int_{\{u=t\}} \frac{w(x)}{|\nabla u|} d \mathcal{H}^{N-1}\right)^{\frac{p-1}{p}} \\
& \geqslant \int_{\{u=t\}} H(\nu) w(x) d \mathcal{H}^{N-1} \\
& =P_{w, H}(\{u>t\} ; \Sigma) \\
& \geqslant c \mu(t)^{\frac{D-1}{D}} .
\end{aligned}
$$

Here, the first inequality is a consequence of Hölder's inequality, the second equality follows from (1.12) (by recalling (3.14) and (5.4) $)$, and the last inequality comes from (5.7). Thus, (5.2) is proved. The characterization of the equality sign in (5.2) follows by noting that the equality holds in (5.2) if and only if the equality sign holds in both the inequalities in (5.8).

We are now ready for the

Proof of Theorem 1.1. We consider the function $K$ defined in (3.8), with $\alpha$ and $\beta$ as in (3.9). Since $K(t)$ is nonnegative and, by item (iv) of Lemma 3.3, nonincreasing, we have that

$$
K\left(0^{-}\right) \geqslant K\left(0^{+}\right)-K\left(M^{-}\right) \geqslant \int_{0}^{M}-K^{\prime}(t) d t .
$$

Combining this inequality with (3.10) leads to

$$
K\left(0^{-}\right) \geqslant \int_{0}^{M}\left[\alpha I(t)^{\alpha-1} \mu(t)^{\beta} f(t)+\beta I(t)^{\alpha} \mu(t)^{\beta-1}\right]\left(-\mu^{\prime}(t)\right) d t \text { for a.e. } t .
$$

Notice that equality here above holds true when $K$ is absolutely continuous. Being the integrand in the righthand side (that is $-K^{\prime}(t)$ ) and $-\mu^{\prime}(t)$ nonnegative (and hence so is also the factor in the square bracket), we can use (3.11) to get

$$
K\left(0^{-}\right) \geqslant \int_{0}^{M}\left[\alpha I(t)^{\alpha-1} \mu(t)^{\beta} f(t)+\beta I(t)^{\alpha} \mu(t)^{\beta-1}\right]\left(\int_{\{u=t\}} \frac{w(x)}{|\nabla u|} d \mathcal{H}^{N-1}\right) d t
$$

Then we compute

$$
\begin{aligned}
K\left(0^{-}\right) & \geqslant \int_{0}^{M}\left[\alpha I(t)^{\alpha-1} \mu(t)^{\beta} f(t)+\beta I(t)^{\alpha} \mu(t)^{\beta-1}\right]\left(\int_{\{u=t\}} \frac{w(x)}{|\nabla u|} d \mathcal{H}^{N-1}\right) d t \\
& =\int_{0}^{M}\left[\alpha I(t)^{\alpha-1-\frac{1}{p-1}} \mu(t)^{\beta} f(t)+\beta I(t)^{\alpha-\frac{1}{p-1}} \mu(t)^{\beta-1}\right] I(t)^{\frac{1}{p-1}}\left(\int_{\{u=t\}} \frac{w(x)}{|\nabla u|} d \mathcal{H}^{N-1}\right) d t \\
& \geqslant \int_{0}^{M} c^{\frac{p}{p-1}}\left[\alpha I(t)^{\alpha-1-\frac{1}{p-1}} \mu(t)^{\beta} f(t)+\beta I(t)^{\alpha-\frac{1}{p-1}} \mu(t)^{\beta-1}\right] \mu(t)^{\frac{p(D-1)}{(p-1) D}} d t
\end{aligned}
$$

where the last inequality follows from (5.2) and the fact that the factor in the square brackets is nonnegative, being $-K^{\prime} \geqslant 0$.

Since (5.10) has been obtained by applying (5.2) on almost all the level sets, a necessary condition to get the equality in (5.10) is that, for a.e. $t \in(0, M),\{u>t\}$ is a minimizer of (4.1) and $H(\nabla u)$ is constant on $\{u=t\}$.

Now we notice that the values of $\alpha$ and $\beta$ in (3.9) are set in order to satisfy

Thus, by setting

$$
\alpha-1-\frac{1}{p-1}=0 \quad \text { and } \quad \beta-1+\frac{p(D-1)}{(p-1) D}=0 \text {. }
$$

$$
F(s):=\int_{0}^{s} f(\tau) d \tau
$$


and $p^{\prime}=p /(p-1)$, (5.10) leads to

$$
\begin{aligned}
K\left(0^{-}\right) & \geqslant \int_{0}^{M} c^{p^{\prime}}\left[p^{\prime} f(t) w(\{u>t\})+\frac{p-D}{D(p-1)} \int_{\{u>t\}} f(u) w(x) d \mathcal{H}^{N}\right] d t \\
& =c^{p^{\prime}} \int_{0}^{M} \int_{\Sigma \cap \Omega} \chi_{\{u>t\}} w(x)\left(p^{\prime} f(t)+\frac{p-D}{D(p-1)} f(u)\right) d \mathcal{H}^{N} d t \\
& =c^{p^{\prime}}\left[p^{\prime} \int_{\Sigma \cap \Omega} F(u) w(x) d \mathcal{H}^{N}+\frac{p-D}{D(p-1)} \int_{\Sigma \cap \Omega} u f(u) w(x) d \mathcal{H}^{N}\right] \\
& =c^{p^{\prime}} \frac{p^{\prime}}{D}\left[D \int_{\Sigma \cap \Omega} F(u) w(x) d \mathcal{H}^{N}+\frac{p-D}{p} \int_{\Sigma \cap \Omega} u f(u) w(x) d \mathcal{H}^{N}\right] .
\end{aligned}
$$

By noting that

$$
K\left(0^{-}\right)=\lim _{t \rightarrow 0^{-}} K(t)=w(\Sigma \cap \Omega)^{\frac{p-D}{D(p-1)}}\left[\int_{\Sigma \cap \Omega} f(u) w(x) d \mathcal{H}^{N}\right]^{p^{\prime}}
$$

we deduce from (5.11) that

$$
\frac{D}{p^{\prime} c^{p^{\prime}}} w(\Sigma \cap \Omega)^{\frac{p-D}{D(p-1)}}\left[\int_{\Sigma \cap \Omega} f(u) w(x) d \mathcal{H}^{N}\right]^{p^{\prime}} \geqslant D \int_{\Sigma \cap \Omega} F(u) w(x) d \mathcal{H}^{N}+\frac{p-D}{p} \int_{\Sigma \cap \Omega} u f(u) w(x) d \mathcal{H}^{N} .
$$

Now we recall the weighted anisotropic Pohozaev identity (3.17):

$$
D \int_{\Sigma \cap \Omega} F(u) w(x) d \mathcal{H}^{N}+\frac{p-D}{p} \int_{\Sigma \cap \Omega} u f(u) w(x) d \mathcal{H}^{N}=\frac{1}{p^{\prime}} \int_{\Gamma_{0}} H^{p}(\nabla u)\langle x, \nu\rangle w(x) d \mathcal{H}^{N-1},
$$

where $\Gamma_{0}=\Sigma \cap \partial \Omega$. By using for the first time that $\Omega$ is a Wulff ball, (2.1) gives that

$$
\langle x, \nu\rangle=R H(\nu) \quad \text { for } x \in \partial \Omega \supseteq \Gamma_{0},
$$

where $R$ is the radius of the Wulff ball $\Omega$, and hence

$$
\frac{1}{p^{\prime}} \int_{\Gamma_{0}} H^{p}(\nabla u)\langle x, \nu\rangle w(x) d \mathcal{H}^{N-1}=\frac{R}{p^{\prime}} \int_{\Gamma_{0}} H^{p}(\nabla u) H(\nu) w(x) d \mathcal{H}^{N-1} .
$$

By using Hölder's inequality and (1.12) we obtain

$$
\begin{aligned}
\int_{\Gamma_{0}} H^{p-1}(\nabla u) H(\nu) w(x) d \mathcal{H}^{N-1} & =\int_{\Gamma_{0}}[H(\nu) w(x)]^{\frac{1}{p}}\left[H^{p-1}(\nabla u)(H(\nu) w(x))^{\frac{1}{p}}\right] d \mathcal{H}^{N-1} \\
& \leqslant P_{w, H}(\Omega ; \Sigma)^{\frac{1}{p}}\left[\int_{\Gamma_{0}} H^{p}(\nabla u) H(\nu) w(x) d \mathcal{H}^{N-1}\right]^{\frac{1}{p^{\prime}}} .
\end{aligned}
$$

As a result, exploiting (5.1) (which also holds true for $t=0$ ), we conclude that

$$
\int_{\Gamma_{0}} H^{p}(\nabla u) H(\nu) w(x) d \mathcal{H}^{N-1} \geqslant \frac{1}{P_{w, H}(\Omega ; \Sigma)^{\frac{1}{p-1}}}\left[\int_{\Sigma \cap \Omega} f(u) w(x) d \mathcal{H}^{N}\right]^{p^{\prime}} .
$$

The last inequality, (5.13) and (3.17) entail that

$$
D \int_{\Sigma \cap \Omega} F(u) w(x) d \mathcal{H}^{N}+\frac{p-D}{p} \int_{\Sigma \cap \Omega} u f(u) w(x) d \mathcal{H}^{N} \geqslant \frac{R}{p^{\prime} P_{w, H}(\Omega ; \Sigma)^{\frac{1}{p-1}}}\left[\int_{\Sigma \cap \Omega} f(u) w(x) d \mathcal{H}^{N}\right]^{p^{\prime}} .
$$

By recalling (5.3) and (2.7), the straightforward computation

$$
\frac{D}{p^{\prime} c^{p^{\prime}}} w(\Sigma \cap \Omega)^{\frac{p-D}{D(p-1)}}=\frac{R}{p^{\prime} P_{w, H}(\Omega ; \Sigma)^{\frac{1}{p-1}}}
$$

shows that (5.12) and (5.14) are opposite inequalities. Thus, they hold with the equality sign, and hence, for a.e. $t \in(0, M),\{u>t\}$ is a is a minimizer of (4.1) and $H(\nabla u)$ is constant on $\{u=t\}$.

Lemma 5.2. Suppose that the assumptions of Theorem 1.1 are satisfied. Up to rotations, we write $\Sigma=$ $\mathbb{R}^{k} \times \tilde{\Sigma}$, where $0 \leqslant k \leqslant N$ and $\tilde{\Sigma} \subset \mathbb{R}^{N-k}$ is an open convex cone containing no lines. Assume that for a.e. $t \in(0, M)$ the following two conditions are verified:

(i) $\{u>t\}=\Sigma \cap B_{\rho(t)}(x(t))$ where $B_{\rho(t)}(x(t))$ is the Wulff ball of radius $\rho(t) \geqslant 0$ centered at the point $x(t) \in \mathbb{R}^{k} \times\left\{0_{\mathbb{R}^{N-k}}\right\}$, with $\rho(t)$ and $x(t)$ depending on $t$;

(ii) $H(\nabla u)$ is constant on $\{u=t\}$. 
Then, $u$ is a radially symmetric and radially nonincreasing function. Moreover,

$$
u \text { is radially strictly decreasing on }\{0<u<M\} \text {, }
$$

and

$$
\{0<u<M\} \quad \text { is a Wulff annulus or a punctured Wulff ball centered at } 0 \text { intersected with } \Sigma \text {. }
$$

Proof. Let us consider the case $k=N$. We follow the ideas used in [Se, Lemma 6] in the isotropic setting. By reasoning as in [Se, Lemma 6], we easily find that

$$
\{u>t\}=B_{\rho(t)}(x(t)) \quad \text { for every } t \in(0, M),
$$

where $B_{\rho(t)}(x(t))$ denotes the Wulff ball centered at $x(t)$ with radius $\rho(t)$. Hence, by continuity also (ii) holds for every $t \in(0, M)$, that is, $H(\nabla u)$ is constant on $\partial B_{\rho(t)}(x(t))$ for every $t \in(0, M)$. By (5.1), we thus find that

$$
P_{w, H}\left(\partial B_{\rho(t)}(x(t))\right) H\left(\nabla u\left(\partial B_{\rho(t)}(x(t))\right)\right)^{p-1}=\int_{B_{\rho(t)}(x(t))} f(u) w(x) d \mathcal{H}^{N} .
$$

Notice that, since $f \geqslant 0$, by the maximum principle we cannot have $f \equiv 0$ on $\{u>t\}$ for $t \in(0, M)$. Hence, $\nabla u$ does not vanish in $\{0<u<M\}$.

Thus, $u$ is a $C^{1}$ function whose gradient never vanishes in $\{u<M\}$, and hence $\widetilde{\mu}(t):=\mathcal{H}^{N}(\{u>t\})$ is locally Lipschitz in $(0, M)$. Therefore, also $\rho(t)=\left(\widetilde{\mu}(t) / \mathcal{H}^{N}(B)\right)^{1 / N}$ is locally Lipschitz, being $\mathcal{H}^{N}(B)$ the volume of the unitary Wulff ball.

We observe that $B_{\rho(t)}(x(t))=\{u \geqslant t\} \supset\{u \geqslant s\}=B_{\rho(s)}(x(s))$ for $t<s$, and therefore

$$
x(s)+\rho(s) \frac{x(s)-x(t)}{H_{0}(x(s)-x(t))} \in B_{\rho(s)}(x(s)) \subset B_{\rho(t)}(x(t)) .
$$

As a consequence,

$$
\begin{aligned}
\rho(t) & \geqslant H_{0}\left(x(s)+\rho(s) \frac{x(s)-x(t)}{H_{0}(x(s)-x(t))}-x(t)\right) \\
& =H_{0}\left(\frac{x(s)-x(t)}{H_{0}(x(s)-x(t))}\left(H_{0}(x(s)-x(t))+\rho(s)\right)\right) \\
& =H_{0}(x(s)-x(t))+\rho(s),
\end{aligned}
$$

where in the last identity we used the homogeneity of $H_{0}$, and accordingly

$$
H_{0}(x(t)-x(s)) \leqslant \rho(t)-\rho(s) \text { for } t<s,
$$

that gives that $x(t)$ is also locally Lipschitz.

Now suppose by contradiction that $u$ is not radially symmetric. Then $x(t)$ would not be identically constant in $(0, M)$ and hence we could find some $t_{0} \in(0, M)$ such that the velocity vector $x^{\prime}\left(t_{0}\right)$ would exist and be nonzero. Set

$$
\bar{\xi}:=\frac{x^{\prime}\left(t_{0}\right)}{H_{0}\left(x^{\prime}\left(t_{0}\right)\right)}, \quad P(t):=x(t)+\rho(t) \bar{\xi} \quad \text { and } \quad Q(t):=x(t)-\rho(t) \bar{\xi} .
$$

By definition, $P(t)$ and $Q(t)$ belong to $\partial\{u>t\}$ for every $t$, and hence we have

$$
\nabla u\left(P\left(t_{0}\right)\right)=-\left|\nabla u\left(P\left(t_{0}\right)\right)\right| \nu\left(P\left(t_{0}\right)\right) \quad \text { and } \quad \nabla u\left(Q\left(t_{0}\right)\right)=-\left|\nabla u\left(Q\left(t_{0}\right)\right)\right| \nu\left(Q\left(t_{0}\right)\right),
$$

where $\nu$ denotes the exterior unit normal vector field to $\left\{u>t_{0}\right\}=B_{\rho\left(t_{0}\right)}\left(x\left(t_{0}\right)\right)$. Moreover, since $\bar{\xi} \in B=$ $\left\{x \in \mathbb{R}^{N}: H_{0}(x)=1\right\}$, by (2.1) it holds that

$$
\left\langle\nu_{B}(\bar{\xi}), \bar{\xi}\right\rangle=H\left(\nu_{B}(\bar{\xi})\right)
$$

We also observe that

$$
\nu\left(P\left(t_{0}\right)\right)=\nu_{B}(\bar{\xi})=-\nu_{B}(-\bar{\xi})=-\nu\left(Q\left(t_{0}\right)\right) .
$$

To check this, we let $B_{r}=\left\{H_{0}(x)<r\right\}$ be the Wulff ball of radius $r$ centered at the origin, and set $B:=B_{1}$. For any $x \in \partial B_{r}$ it holds that

$$
\nu_{B_{r}}(x)=\frac{\nabla_{x} H_{0}(x)}{\left|\nabla_{x} H_{0}(x)\right|} \quad \text { and } \quad \nu_{B_{r}}(-x)=\frac{\nabla_{x} H_{0}(-x)}{\left|\nabla_{x} H_{0}(-x)\right|} .
$$

Also, by the homogeneity of $H_{0}$ we have that

$$
\nabla_{x} H_{0}(t x)=\operatorname{sgn}(t) \nabla_{x} H_{0}(x) \text { for all } t \neq 0, x \neq 0
$$

and consequently

$$
\nu_{B_{r}}(r \xi)=\nu_{B}(\xi)=-\nu_{B}(-\xi)=\nu_{B_{r}}(-r \xi) \quad \text { for any } \xi \in \partial B, r \in \mathbb{R}^{+} .
$$


Additionally, taking into account the translation invariance of the normals, we obtain

$$
\nu_{B_{r}(x)}(x+r \xi)=\nu_{B_{r}}(r \xi) \quad \text { for any } x \in \mathbb{R}^{N}, r \in \mathbb{R}^{+}, \xi \in \partial B .
$$

Combining this information and (5.20) we obtain (5.19), as desired.

Thus, from (5.18) and (5.19) we deduce that

$$
\left\langle\nu\left(P\left(t_{0}\right)\right), \bar{\xi}\right\rangle=H\left(\nu\left(P\left(t_{0}\right)\right)\right) \quad \text { and } \quad\left\langle\nu\left(Q\left(t_{0}\right)\right), \bar{\xi}\right\rangle=-H\left(\nu\left(Q\left(t_{0}\right)\right)\right) .
$$

From this and (5.17), it follows that

$$
\left\langle\nabla u\left(P\left(t_{0}\right)\right), \bar{\xi}\right\rangle=-H\left(\nabla u\left(P\left(t_{0}\right)\right)\right) \quad \text { and } \quad\left\langle\nabla u\left(Q\left(t_{0}\right)\right), \bar{\xi}\right\rangle=H\left(\nabla u\left(Q\left(t_{0}\right)\right)\right) .
$$

By using (5.16) and (5.21), we compute

$$
1=\left.\frac{d}{d t} u(P(t))\right|_{t=t_{0}}=\left\langle\nabla u\left(P\left(t_{0}\right)\right),\left(H_{0}\left(x^{\prime}\left(t_{0}\right)\right)+\rho^{\prime}\left(t_{0}\right)\right) \bar{\xi}\right\rangle=-H\left(\nabla u\left(P\left(t_{0}\right)\right)\right)\left(H_{0}\left(x^{\prime}\left(t_{0}\right)\right)+\rho^{\prime}\left(t_{0}\right)\right)
$$

and

$$
1=\left.\frac{d}{d t} u(Q(t))\right|_{t=t_{0}}=\left\langle\nabla u\left(Q\left(t_{0}\right)\right),\left(H_{0}\left(x^{\prime}\left(t_{0}\right)\right)-\rho^{\prime}\left(t_{0}\right)\right) \bar{\xi}\right\rangle=H\left(\nabla u\left(Q\left(t_{0}\right)\right)\right)\left(H_{0}\left(x^{\prime}\left(t_{0}\right)\right)-\rho^{\prime}\left(t_{0}\right)\right) .
$$

But by assumption (ii), we have that $H\left(\nabla u\left(P\left(t_{0}\right)\right)\right)=H\left(\nabla u\left(Q\left(t_{0}\right)\right)\right)$. This leads to $H_{0}\left(x^{\prime}\left(t_{0}\right)\right)=0$, which is a contradiction.

Thus, $u$ is radially symmetric, i.e., $u=u(r)$, with $r=H_{0}(x)$. Since we showed that $\nabla u$ does not vanish on $\{0<u<M\}$, we thus have $\partial_{r} u<0$ in this open ring. The set $\{u=M\}$ could be a point, but also a closed ball (as it happens, e.g., in the example described in [Se, pag. 1895]). This proves the result when $k=N$.

By direct inspection and recalling that $u$ is $C^{1}$ up to $\Gamma_{1} \backslash\{0\}$, it is easy to check that the same proof remains valid when $1 \leqslant k \leqslant N-1$. Notice that in this case we have that $\bar{\xi} \in \mathbb{R}^{k} \times\left\{0_{\mathbb{R}^{N-k}}\right\}$.

The case $k=0$ is trivial.

We have now all the ingredients to complete the

Proof of Theorems 1.4, 1.5 and 1.6. Theorems 1.4 and 1.5 easily follow by putting together Theorems 1.1 . 4.2 , and Lemma 5.2

Theorem 1.6 easily follows by putting together Theorems 1.1, 4.3, and Lemma 5.2.

\section{ACKNOWLEDGEMENTS}

The authors are members of INdAM/GNAMPA and AustMS and are supported by the Australian Research Council Discovery Project DP170104880 NEW "Nonlocal Equations at Work". The first author is supported by the Australian Research Council DECRA DE180100957 "PDEs, free boundaries and applications". The second and third authors are supported by the Australian Laureate Fellowship FL190100081 "Minimal surfaces, free boundaries and partial differential equations".

The authors thank Xavier Cabré for bringing up to their attention the reference [Se]. The authors also thank Xavier Ros-Oton and Joaquim Serra for their useful comments on a preliminary version of this paper, Giulio Ciraolo for the helpful correspondence about the forthcoming article [ACF], and Emanuel Indrei for kindly pointing out his recent results in [I].

\section{REFERENCES}

[A] A.D. Aleksandrov, Uniqueness theorems for surfaces in the large. V, Vestnik Leningrad. Univ. 13 1958 no. 19, 5-8.

[AB] M. Amar, G. Bellettini, A notion of total variation depending on a metric with discontinuous coefficients, Ann. Inst. H. Poincaré Anal. Non Linéaire 11 (1994), no. 1, 91-133.

[AFP] L. Ambrosio, N. Fusco, D. Pallara, Functions of bounded variation and free discontinuity problems. Oxford Mathematical Monographs. The Clarendon Press, Oxford University Press, New York, 2000.

[ACF $] \quad$ C.A. Antonini, G. Ciraolo, and A. Farina, Interior regularity results for anisotropic quasilinear equations, forthcoming.

$[\mathrm{BBF}] \quad$ G. Bellettini, G. Bouchitté, and I. Fragalà, BV functions with respect to a measure and relaxation of metric integral functionals, J. Convex Anal. 6 (1999), 349-366.

[BFK] M. Belloni, V. Ferone, and B. Kawohl, Isoperimetric inequalities, Wulff shape and related questions for strongly nonlinear elliptic operators, Special issue dedicated to Lawrence E. Payne. Z. Angew. Math. Phys. 54 (2003), no. 5, $771-783$.

[BP] H. Berestycki, F. Pacella, Symmetry properties for positive solutions of elliptic equations with mixed boundary conditions, J. Funct. Anal. 87 (1989), 177-211.

[BC] C. Bianchini, G. Ciraolo, Wulff shape characterizations in overdetermined anisotropic elliptic problems, Commun. Partial Differ. Equ. 43 (2018), 79-820.

[BNST] B. Brandolini, C. Nitsch, P. Salani, and C. Trombetti, Serrin type overdetermined problems: an alternative proof, Arch. Rat. Mech. Anal.190(2008), 267-280.

[BM] J.E. Brothers, F. Morgan, The isoperimetric theorem for general integrands, Michigan Math. J.41 (1994), no. 3, $419-431$.

[BZ] J.E. Brothers, W.P. Ziemer, Minimal rearrangements of Sobolev functions, J. Reine Angew. Math. 384 (1988), 153179. 
[CRS] X. Cabré, X. Ros-Oton, and J. Serra, Sharp isoperimetric inequalities via the ABP method, J. Eur. Math. Soc. (JEMS) 18 (2016), no. 12, 2971-2998.

[CR] A. Cañete, C. Rosales, Compact stable hypersurfaces with free boundary in convex solid cones with homogeneous densities, Calc. Var. Partial Differential Equations 51 (2014), 887-913.

[CDLP] M. Carriero, G. Dal Maso, A. Leaci, and E. Pascali: Relaxation of the non-parametric Plateau problem with an obstacle, J. Math. Pures Appl. 67, 359-396 (1988).

[Ch] G.R. Chambers, Proof of the log-convex density conjecture, J. Eur. Math. Soc. (JEMS) 21 (2019), no. 8, $2301-2332$.

[CM] A. Cianchi, V. Maz'ya, Global Lipschitz regularity for a class of quasilinear elliptic equations, Comm. Part. Diff. Equat. 36 (2011), 100-133.

[CS] A. Cianchi, P. Salani, Overdetermined anisotropic elliptic problems, Math. Ann. 345 (2009), 859-881.

[CGPRS] E. Cinti, F. Glaudo, A. Pratelli, X. Ros-Oton, and J. Serra, Sharp quantitative stability for isoperimetric inequalities with homogeneous weights, arXiv:2006.13867v1.

[CFR] G. Ciraolo, A. Figalli, A. Roncoroni, Symmetry results for critical anisotropic p-Laplacian equations in convex cones, Geom. Funct. Anal., 30 (2020), 770-803.

[CG] G. Ciraolo, A. Greco, An overdetermined problem associated to the Finsler Laplacian, Commun. Pure Appl. Anal., 2021, $20(3): 1025-1038$.

[CiR] G. Ciraolo, A. Roncoroni, Serrin's type overdetermined problems in convex cones, Calc. Var. Partial Differential Equations 59 (2020), no. 1, 21 pp.

[CFV] M. Cozzi, A. Farina, and E. Valdinoci, Monotonicity formulae and classification results for singular, degenerate, anisotropic PDEs, Adv. Math. 293 (2016), 343-381.

[CrM] G. Crasta, A. Malusa, The distance function from the boundary in a Minkowski space, Trans. Amer. Math. Soc. 359 (2007), no. 12, 5725-5759.

[DP] B. Dacorogna, C.E. Pfister, Wulff theorem and best constant in Sobolev inequality, J. Math. Pures Appl.(9) 71 (2) (1992), 97-118.

[DMS] M. Degiovanni, A. Musesti, and M. Squassina, On the regularity of solutions in the Pucci-Serrin identity, Calc. Var. Partial. Differ. Equations 18 (2003), 317-334.

[Di] A. Dinghas, Über einen geometrischen Satz von Wulff für die Gleichgewichtsform von Kristallen, Z. Kristallogr., Mineral. Petrogr. 105, (1944).

[DPV] S. Dipierro, G. Poggesi, and E. Valdinoci, A Serrin-type problem with partial knowledge of the domain, Nonlinear Analysis 208 (2021), 112330.

[DFM] J. Dolbeault, P. Felmer, and R. Monneau, Symmetry and nonuniformly elliptic operators, Differential Integral Equations 18 (2005), no. 2, 141-154.

[EP] A. Enciso, D. Peralta-Salas, Symmetry for an overdetermined boundary problem in a punctured domain, Nonlinear Anal. 70 (2009), no. 2, 1080-1086.

[EG] L.C. Evans, R.F. Gariepy, Measure Theory and Fine Poperties of Functions, Studies in Advanced Mathematics, CRC Press, 1992.

[FK] A. Farina, B. Kawohl, Remarks on an overdetermined boundary value problem, Calc. Var. Partial Differential Equations 31(2008), no. 3, 351-357.

[Fe] H. Federer, Curvature measures, Trans. Amer. Math. Soc. 93 (1959), 418-491.

[FI] A. Figalli, E. Indrei, A sharp stability result for the relative isoperimetric inequalityinside convex cones, J. Geom. Anal. 23 (2013), no. 2, 938-969.

[FMP] A. Figalli, F. Maggi, and A. Pratelli, A mass transportation approach to quantitative isoperimetric inequalities, Invent. Math. 182 (2010), no. 1, 167-211.

[FM] I. Fonseca, S. Müller, A uniqueness proof for the Wulff theorem, Proc. Roy. Soc. Edinburgh Sect. A 119 (1991), $125-136$.

[FGK] I. Fragalà, F. Gazzola, and B. Kawohl, Overdetermined problems with possibly degenerate ellipticity, a geometric approach, Math. Z. 254(2006), 117-132.

[GL] N. Garofalo, J.L. Lewis, A symmetry result related to some overdetermined boundary value problems, Amer. J. Math. 111 (1989), no. 1, 9-33.

[GS] N. Garofalo, E. Sartori, Symmetry in exterior boundary value problems for quasilinear elliptic equations via blow-up and a-priori estimates, Adv. Differential Equations 4 (1999), 137-161.

[GNN] B. Gidas, W.M. Ni, and L. Nirenberg, Symmetry and related properties via the maximum principle, Comm. Math. Phys. 68 (1979), no. 3, 209-243.

[GT] D. Gilbarg, N.S. Trudinger, Elliptic partial differential equations of second order, Classics in Mathematics, SpringerVerlag, Berlin, 2001. Reprint of the 1998 edition.

[Gi] E. Giusti, Minimal surfaces and functions of bounded variation, Birkhäuser, Boston, 1984.

[GX] J. Guo, G. Xia, A partially overdetermined problem in a half ball, Calc. Var. Partial Differential Equations 58 (2019), no. 5, Art. 160, 1-15.

[H] J. Heinonen, Lectures on Lipschitz analysis. No. 100. University of Jyväskylä, 2005.

[I] E. Indrei, A weighted relative isoperimetric inequality in convex cones, Methods Appl. Anal. 28 (2021), no. 1, 1-13.

[KP] S. Kesavan, F. Pacella, Symmetry of positive solutions of a quasilinear elliptic equation via isoperimetric inequalities, Appl. Anal. 54 (1994), no. 1-2, 27-37.

[L] P.-L. Lions, Two geometrical properties of solutions of semilinear problems, Applicable Anal. 12 (1981), no. 4, 267-272.

[LP] P.-L. Lions, F. Pacella, Isoperimetric inequalities for convex cones, Proc. Amer. Math. Soc. 109 (1990), no. 2, $477-485$.

[Li] M. Lieberman, Boundary regularity for solutions of degenerate elliptic equations, Nonlinear Anal. 12 (1988), 12031219 .

[Lo] H. Lou, On singular sets of local solutions to p-Laplace equations, Chin. Ann. Math. 29B(5) (2008), 521-530.

[Ma] F. Maggi, Sets of finite perimeter and geometric variational problems: an introduction to Geometric Measure Theory, Cambridge University Press, 2012.

[MP] R. Magnanini, G. Poggesi, On the stability for Alexandrov's Soap Bubble theorem, J. Anal. Math., 139(1):179-205, 2019. 
[MP2] R. Magnanini, G. Poggesi, Serrin's problem and Alexandrov's Soap Bubble Theorem: enhanced stability via integral identities, Indiana Univ. Math. J. 69 (2020), no. 4, 1181-1205.

[MP3] R. Magnanini, G. Poggesi, Nearly optimal stability for Serrin's problem and the Soap Bubble theorem, Calc. Var. Partial Differential Equations, 59(1):Paper No. 35, 2020.

[MR] E. Milman, L. Rotem, Complemented Brunn-Minkowski inequalities and isoperimetry for homogeneous and nonhomogeneous measures, arXiv:1308.5695v2.

[MS] V.D. Milman, G. Schechtman, Asymptotic theory of finite-dimensional normed spaces. With an appendix by M.Gromov. Lecture Notes in Mathematics, 1200. Springer-Verlag, Berlin, 1986. viii+156 pp.

[Mo] F. Morgan, The log-convex density conjecture, Frank Morgan's blog (April 2010), http://sites.williams.edu/Morgan/2010/04/03/the-log-convex-density-conjecture/

[PT] F. Pacella, G. Tralli, Overdetermined problems and constant mean curvature surfaces in cones, Rev. Mat. Iberoam. 36 (2020), 841-867.

[PT2] F. Pacella, G. Tralli, Isoperimetric cones and minimal solutions of partial overdetermined problems, Publ. Mat. 65(1): 61-81 (2021).

[PSc] L.E. Payne, P.W. Schaefer, Duality theorems in some overdetermined problems, Math. Methods Appl. Sci. 11 (1989), no. 6, 805-819.

[Po] G. Poggesi, Radial symmetry for p-harmonic functions in exterior and punctured domains, Appl. Anal. 98 (2019), no. 10, 1785-1798.

[PS] P. Pucci, J. Serrin, A general variational identity Indiana Univ. Math. J. 35 (1986), 681-703.

[Re] R. C. Reilly, Applications of the Hessian operator in a Riemannian manifold, Indiana Univ. Math. J. 26 (1977), 459-472.

[Se] J. Serra, Radial symmetry of solutions to diffusion equation with discontinuous nonlinearities, J. Differential Equations 254 (2013), 1893-1902.

[S] J. Serrin, A symmetry problem in potential theory, Arch. Rational Mech. Anal. 43 (1971), $304-318$.

[Sa] T. Sakai, Riemannian geometry, Translations of Mathematical Monographs, vol. 149, American Mathematical Society, Providence, RI, 1996.

[Scha] J. Van Schaftingen, Anisotropic symmetrization, Ann. Inst. H. Poincaré Anal. Non Linéaire 23 (2006), no. 4, $539-565$.

[Sc] R. Schneider, Convex Bodies: The Brunn-Minkowski Theory, Cambridge University Press, Cambridge (1993).

[Tal] G. Talenti, A weighted version of a rearrangement inequality. Ann. Univ. Ferrara Sez. VII (N.S.) 43 (1997), 121-133 (1998).

[Ta] J. Taylor, Crystalline variational problems, Bull. Amer. Math. Soc. 84 (1978), 568-588.

[To] P. Tolksdorf, Regularity for a more general class of quasilinear elliptic equations, J. Diff. Eq. 51 (1984), $126-150$.

[WX] G. Wang, C. Xia. A characterization of the Wulff shape by an overdetermined anisotropic PDE, Arch. Rational Mech. Anal. 199 (2011) 99-115.

[W] H. F. Weinberger, Remark on the preceding paper of Serrin, Arch. Rational Mech. Anal. 43 (1971), 319-320.

Serena Dipierro: Department of Mathematics and Statistics, The University of Western Australia, 35 Stirling Highway, Crawley, Perth, WA 6009, Australia

Email address: serena.dipierro@uwa.edu.au

Giorgio Poggesi: Department of Mathematics and Statistics, The University of Western Australia, 35 Stirling Highway, Crawley, Perth, WA 6009, Australia

Email address: giorgio.poggesi@uwa.edu.au

Enrico Valdinoci: Department of Mathematics and Statistics, The University of Western Australia, 35 Stirling Highway, Crawley, Perth, WA 6009, Australia

Email address: enrico.valdinoci@uwa.edu.au 